\title{
On the Friedlander-Milnor conjecture for groups of small rank
}

Fabien Morel

\section{Contents}

0. Introduction

1. The $\mathbb{A}^{1}$-local space $B \operatorname{Sing} \mathbb{A}^{1}(G)$

1.1. The Suslin-Voevodsky construction 55

1.2. The $\mathbb{A}^{1}$-local space $\operatorname{Sing}_{\bullet}^{\mathbb{A}^{1}}(G)$ and its classifying space 56

2. The Friedlander conjecture and the rigidity property 57

2.1. The rigidity properties at $\ell \quad 57$

2.2. Reformulation of the rigidity property in term of simplicial chain complex 60

3. The rigidity property and the $\mathbb{A}^{1}$-chain complex 64

3.1. $\mathbb{A}^{1}$-chain complexes and the $\mathbb{A}^{1}$-lower central series 64

3.2. $\mathbb{A}^{1}$-homological characterization of the rigidity property 68

4. Reduction to the rigidity property for the $C_{*}^{\mathbb{A}^{1}}\left(\left(\mathbb{G}_{m}\right)^{\wedge n}\right)^{\prime} \mathrm{s} \quad 71$

4.1. $\mathbb{A}^{1}$-chain complex of mixed Tate type $\quad 71$

4.2. Bruhat decomposition and $\mathbb{A}^{1}$-chain complex of $G \quad 73$

5. The rigidity property for the $C_{*}^{\mathbb{A}^{1}}\left(\left(\mathbb{G}_{m}\right)^{\wedge n}\right)^{\prime}$ s $\quad 76$

5.1. The Transfers morphisms on the $\mathbb{A}^{1}$-localization of the $\mathbb{Z}\left(\mathbb{G}_{m}^{\wedge n}\right)$ 's 76

5.2. Transfers structures on the $H_{*}^{\mathbb{A}^{1}}\left(\left(\mathbb{G}_{m}\right)^{n}\right)$ 's and rigidity $\quad 82$

A $\quad \mathbb{A}^{1}$-coverings and Friedlander conjecture in degree $2 \quad 86$

A.1. $\quad \mathbb{A}^{1}$-coverings and central extensions of algebraic groups $\quad 87$

A.2. $\quad$ Proof of the Friedlander conjecture in degree $2 \quad 89$

$\begin{array}{ll}\text { References } & 91\end{array}$

(C) 2011 International Press 


\section{Introduction}

The title refers to the following conjecture of E. Friedlander [12]:

CONJECTURE 1. For any algebraically closed field $F$, any reductive algebraic $F$-group $G$ and any prime $\ell$ different from char $(F)$ the natural ring homomorphism

$$
H_{e t}^{*}(B G ; \mathbb{Z} / \ell) \rightarrow H^{*}(B G(F) ; \mathbb{Z} / \ell)
$$

from the $\mathbb{Z} / \ell$-étale cohomology of the simplicial classifying space of $G$ to the cohomology with $\mathbb{Z} / \ell$-coefficients of the discrete group $G(F)$ is an isomorphism.

and with J. Milnor's variant in the case $F=\mathbb{C}[\mathbf{3 5}]$ :

Conjecture 2. For any Lie group $G$ and any prime $\ell$ the natural ring homomorphism

$$
H_{\text {sing }}^{*}(B G ; \mathbb{Z} / \ell) \rightarrow H^{*}\left(B G^{\delta} ; \mathbb{Z} / \ell\right)
$$

from the $\mathbb{Z} / \ell$-singular cohomology of the classifying space $B G$ of the topological group $G$ to the $\mathbb{Z} / \ell$-singular cohomology of the classifying space $B G^{\delta}$ of the discrete group $G^{\delta}$ underlying $G$ is an isomorphism.

The two conjectures are equivalent for $F=\mathbb{C}$ and $G$ a complex algebraic Lie group by Grothendieck's comparisons theorems between étale and singular cohomology, but each contains cases which are not reached by the other.

The aim of these conjectures is the computation of the right hand side that is to say the mod $\ell$ cohomology of the (classifying space of the) discrete group $G(F)$. Indeed, again by Grothendieck's comparisons theorems the left hand side of conjecture 1 is isomorphic to the singular mod $\ell$ cohomology of the topological classifying space of the complex algebraic Lie group $G(\mathbb{C})$ of complex points of $G$. The singular cohomology of the classifying space of a Lie group is in practice already computed (in most cases) by standard technics of algebraic topology and should be considered as "simpler". For instance $H_{\text {sing }}^{*}\left(B S L_{n}(\mathbb{C}) ; \mathbb{Z} / \ell\right)$ is known to be a polynomial ring with coefficients in $\mathbb{Z} / \ell$ on the Chern classes $c_{2}, \ldots, c_{n}$.

The main evidence for these conjectures is the result of Suslin $[\mathbf{6 0}, \mathbf{6 1}]$ which establish the case $G=S L_{\infty}$ (which is not an algebraic group!) for each separably closed field $F$. Friedlander and Mislin also proved the conjecture for $G$ arbitrary and $F$ the algebraic closure of a finite field [14]. Using Suslin's results [62] on stability properties of the homology for $S L_{n}$ one may deduce the conjecture for $S L_{n}$ in (co-)homological degree * small enough compared to $n$. For the classical groups like the orthogonal and symplectic group, one may as well deduce from Suslin Theorem partial results using stability properties; see for instance [29]. Except these cases obtained from Suslin's result by stabilization properties, the conjecture is basically unknown. For further references the reader should check [30] and [50]. Up 
to now in particular, there is no known split simple algebraic group $G$ for which the conjecture holds over any separably closed field $F$.

Let us start with the following result which establishes what we call "the weak Friedlander-Milnor conjecture":

THEOREM 3. Let $F$ be a separably closed field, let $G$ be any reductive algebraic $F$-group $G$ and let $\ell$ be a prime different from char $(F)$. Then the natural homomorphism

$$
H_{e t}^{*}(B G ; \mathbb{Z} / \ell) \rightarrow H^{*}\left(B\left(G\left(\Delta_{F}^{\bullet}\right)\right) ; \mathbb{Z} / \ell\right)
$$

is an isomorphism.

Here $\Delta_{F}^{\bullet}$ denotes the cosimplicial affine $F$-scheme defined by $\Delta_{F}^{n}:=$ $\operatorname{Spec}\left(F\left[T_{0}, \ldots, T_{n}\right] /\left(\sum_{i} T_{i}-1\right)\right)$ and with the standard cosimplicial structure. In the previous Theorem $G\left(\Delta_{F}^{\bullet}\right)$ denotes the simplicial group obtained by evaluating $G$ on $\Delta_{F}^{\bullet}$, and $B G\left(\Delta_{F}^{\bullet}\right)$ means its classifying space (as in [9] for instance). Thus the right hand side of the previous isomorphism is the mod $\ell$ singular cohomology of classifying space of the simplicial group $G\left(\Delta_{F}^{\bullet}\right)$. The morphism itself comes from a commutative square of the form

$$
\begin{aligned}
\left.H_{e t}^{*}\left(B \operatorname{Sing}_{\bullet}^{\mathbb{A}^{1}}(G)\right) ; \mathbb{Z} / \ell\right) & \rightarrow H^{*}\left(B\left(G\left(\Delta_{F}^{\bullet}\right)\right) ; \mathbb{Z} / \ell\right) \\
\downarrow & \\
H_{e t}^{*}(B G ; \mathbb{Z} / \ell) & \rightarrow H^{*}(B(G(F)) ; \mathbb{Z} / \ell)
\end{aligned}
$$

in which $\operatorname{Sing}_{\bullet}^{\mathbb{A}^{1}}(G)$ is the Suslin-Voevodsky construction on $G$ (see below), the left vertical morphism is an isomorphism and the bottom horizontal morphism is the one appearing in the Friedlander conjecture. This diagram will be discussed in Section 1.1 below.

REMARK 4. Classical devissage methods and Theorems for the structure of algebraic groups over perfect fields allow one to extends the previous Theorem to smooth $F$-groups scheme which can be defined over a perfect subfield of $F$. The cases where $G$ is an abelian variety, a smooth unipotent group defined over a perfect subfield, a discrete finite group, or a split Torus are straightforward to check. Thus the main point of the Theorem and of the Friedlander-Milnor conjecture is the case where $G$ is (semi-)simple.

The following property depending on the smooth $F$-group $G$ plays an important role in relating Theorem 3 to the Friedlander conjecture through the above diagram:

Definition 5. Assume $F$ is an infinite field. Let $G$ be a smooth algebraic $F$-group and let $\ell$ be any prime different from $\operatorname{char}(F)$. One says that $G$ satisfies the homotopy invariance of group cohomology at $\ell$ if for any integer $n \geq 1$ the natural ring homomorphism

$$
H^{*}\left(B G\left(F\left[T_{1}, \ldots, T_{n}\right]\right) ; \mathbb{Z} / \ell\right) \rightarrow H^{*}(B G(F) ; \mathbb{Z} / \ell)
$$

is an isomorphism. 
We observe (see [30, page 110]) that if $F$ is finite the homotopy invariance of group cohomology is false in general. The previous notion is relevant because of the following consequence:

Corollary 6. Let $F$ be a separably closed field, let $G$ be any reductive algebraic $F$-group and $\ell$ be a prime different from char $(F)$. If $G$ satisfies the homotopy invariance of group cohomology at $\ell$ then the map of simplicial sets:

$$
B G(F) \rightarrow B\left(G\left(\Delta_{F}^{\bullet}\right)\right)
$$

induces an isomorphism on mod $\ell$-cohomology and consequently the Friedlander conjecture holds for $G$.

Proof. This follows by using the spectral sequences of the bisimplicial set $B G\left(\Delta_{F}^{\bullet}\right)$ of the form

$$
E_{1}^{p, q}=H^{q}\left(B G\left(\Delta_{F}^{p}\right) ; \mathbb{Z} / \ell\right) \Rightarrow H^{p+q}\left(B G\left(\Delta_{F}^{\bullet}\right) ; \mathbb{Z} / \ell\right)
$$

together with the fact that each of the $F$-scheme $\Delta_{F}^{p}$ is an affine $F$-space of dimension $p$.

From [24] it follows that the group $S L_{2}$ doesn't have the property of homotopy invariance of group cohomology at $\ell$. The weak FriedlanderMilnor conjecture reduces the Friedlander-Milnor conjecture for $S L_{2}$ to the statement that the morphism

$$
B S L_{2}(F) \rightarrow B\left(S L_{2}\left(\Delta_{F}^{\bullet}\right)\right)
$$

induces an isomorphism on mod $\ell$ homology for any separably closed field $F$ of characteristic $\neq \ell$.

The $S L_{2}$ case should be quite special and we believe that each semisimple group, not containing the type $S L_{2}$, should satisfy the homotopy invariance of group cohomology at $\ell$, at least over a separably closed field $F$ of characteristic $\neq \ell$.

For the group $S L_{3}$ the homotopy invariance for group cohomology is proven in [30, Theorem 4.6.8] because for polynomial rings with coefficients in a field $F$ one knows that $E_{3}$ agrees with $S L_{3}$ by results of Suslin [59]. The Theorem of Knudson is an elaboration on the work [56] of Soulé who proved the invariance property for each split semi-simple groups in the case of polynomial rings in one variable. In [70] the technics of Knudson are generalized to any split semi-simple group of rank 2. Moreover Wendt also proved in [71] that $S L_{4}$ has the homotopy invariance of group cohomology at $\ell$. Consequently we get altogether:

TheOREM 7. Let $F$ be a separably closed field and let $\ell$ be a prime different from char $(F)$. Let $G$ be a split simple algebraic $F$-group of one of the following type: $S L_{3}, S O_{5}$ or $G_{2}$ and $S L_{4}$. Then the natural homomorphism

$$
H_{e t}^{*}(B G ; \mathbb{Z} / \ell) \rightarrow H^{*}(B G(F) ; \mathbb{Z} / \ell)
$$

is an isomorphism. 
REMARK 8. In a work still in progress, we hope to fix the case of $S L_{2}$ as well by establishing that (0.1) induces an isomorphism on mod $\ell$ homology for any separably closed field $F$ of characteristic $\neq \ell$.

When $F$ is the field of complex numbers, Grothendieck's comparison theorems give some cases of Milnor's form of the conjecture for complex algebraic Lie groups:

COROllary 9. For any complex algebraic Lie group $G$ and any prime $\ell$ the natural ring homomorphism

$$
H_{\text {sing }}^{*}(B G ; \mathbb{Z} / \ell) \rightarrow H^{*}\left(B G\left(\Delta_{\mathbb{C}}^{\bullet}\right)^{\delta} ; \mathbb{Z} / \ell\right)
$$

is an isomorphism. Here $G\left(\Delta_{\mathbb{C}}^{n}\right)^{\delta}$ means the discrete group of algebraic morphisms $\Delta_{\mathbb{C}}^{n} \rightarrow G$, and $B G$ means the classifying space of the topological group underlying $G$. Consequently the morphism

$$
H^{*}(B G ; \mathbb{Z} / \ell) \rightarrow H^{*}\left(B G^{\delta} ; \mathbb{Z} / \ell\right)
$$

is an isomorphism if $G$ is a complex simple algebraic group of type $S L_{3}$, $\mathrm{SO}_{5}, \mathrm{G}_{2}$ or $S L_{4}$.

In Section A of the Appendix we give a direct treatment of the degree 2 case of the Friedlander conjecture, which relies on standard facts on central extensions of algebraic groups and the theory of $\mathbb{A}^{1}$-universal coverings developed in [41]. This should be compared to the results of [50]. Using a slight elaboration of the technics of this work, one may in fact go one step further and prove the conjecture for $* \leq 3$ for any group.

To explain our strategy for the weak Friedlander conjecture, alias Theorem 3 , let us recall that the Quillen algebraic K-theory groups $K_{n}(F)$ of a field $F$ (or of a regular ring) are isomorphic in degree $n \geq 1$ to the KaroubiVillamayor K-theory groups $K_{n}^{K V}(F):=\pi_{n}\left(B\left(\mathbb{G} L_{\infty}\left(\Delta_{F}^{\bullet}\right)\right)\right.$, see for instance [17]. The argument of Suslin relies on the existence of certain transfers morphisms in $\bmod \ell$ algebraic K-theory groups, which are used in a fundamental way to prove the famous rigidity theorem.

Our approach will use extensively the Suslin-Voevodsky construction on $G$, denoted by $\operatorname{Sing} \mathbb{A}^{\mathbb{A}^{1}}(G)$, that is to say the previous construction, but really considered as a simplicial sheaf of groups

$$
\operatorname{Sm}_{k} \ni X \mapsto \operatorname{Sing}_{\bullet}^{\mathbb{A}^{1}}(G)(X):=G\left(\Delta_{k}^{\bullet} \times X\right)
$$

on the category $S m_{k}$ of smooth $k$-schemes in the Nisnevich topology, where $k$ is a fixed perfect base field (like a prime field for instance). Recall $[\mathbf{4 7}, \mathbf{4 4}]$ that the Nisnevich topology has for covering families $\left\{U_{i} \rightarrow X\right\}_{i}$ in $S m_{k}$ the étal covering families with the property that for any point $x \in X$ there is an $i$ and a point $y$ in $U_{i}$ lying over $x$ with the same residue field. Except otherwise stated, in this text we only use the Nisnevich topology on $S m_{k}$.

To be more precise on our approach, we will study the "A ${ }^{1}$-homotopy type" of its classifying space $B \operatorname{Sing} \mathbb{A}_{\bullet}^{1}(G)$, a simplicial sheaf of sets on $S m_{k}$ in 
the Nisnevich topology, or a space in short, see [44]. The main obstruction to adapt Suslin's method to the space $B \operatorname{Sing}_{\bullet}^{\mathbb{A}^{1}}(G)$ comes from the fact that the correspondence $F \mapsto \pi_{n}\left(B \operatorname{Sing}_{\bullet}^{\mathbb{A}^{1}}(G)(F)\right)$ doesn't form a cohomology theory and that there is no way, a priori, to endow it with suitable transfers, as opposed to the above case $G=S L_{\infty}$.

To get round this difficulty we will introduce a rather new approach based on $\mathbb{A}^{1}$-homotopy theory which proceeds roughly speaking in two fundamental steps.

The first step reduces the Theorem 2 to checking a rigidity property for the $\mathbb{A}^{1}$-chain complexes $C_{*}^{\mathbb{A}^{1}}\left(\left(\mathbb{G}_{m}\right)^{\wedge n} ; \mathbb{Z}\right)$ of the smash-powers of $\mathbb{G}_{m}$ 's for all $n$ 's. This reduction step is highly of homotopical nature, but also uses in an essential the Bruhat decomposition for split groups, and also relies essentially on some highly non trivial results from [41]. These results, and part of their proof, contains, on the other hand, the "genes" of the notion of sheaves with generalized transfers which is used in the second step.

Observe that as a consequence, the first step reduces Theorem 2 to a statement which doesn't depend on any group $G$, nor any field $F$.

The second step is to prove the expected rigidity property for these chain complexes $C_{*}^{\mathbb{A}^{1}}\left(\left(\mathbb{G}_{m}\right)^{\wedge n} ; \mathbb{Z}\right)$ : one has to understand why applying the "abstract" $\mathbb{A}^{1}$-localization functor for chain complexes to $\mathbb{Z}\left(\left(\mathbb{G}_{m}\right)^{\wedge n}\right)$ yields a chain complex whose homology sheaves have the rigidity property at $\ell$; this is done by observing that these homology sheaves are endowed with a structure of sheaves with generalized transfers, for which the one considered by Voevodsky [67] are one of the examples. We conclude by a reduction at the very end to the classical rigidity Theorem of $[63]$.

Let us give more details. From now on $k$ is a fixed perfect field.

In Section 1 we give some recollection on the characterization of $\mathbb{A}^{1}$ local spaces $[44,41]$ in terms of $\mathbb{A}^{1}$-homotopy sheaves; this is one of the result that we use from $[\mathbf{4 1}]$. We then prove that the classifying space $\operatorname{BSing} \mathbb{A}^{\mathbb{A}^{1}}(G)$ is $\mathbb{A}^{1}$-local for any split semi-simple group. It is moreover simply connected if $G$ is simply-connected in the sense of algebraic group theory.

In section 2 we observe along the classical lines, see $[\mathbf{6 0}, \mathbf{6 3}, \mathbf{2 8}, \mathbf{3 0}]$, that Theorem 3 is a consequence of the "rigidity property at $\ell$ " of the space $B \operatorname{Sing} g_{\bullet}^{\mathbb{A}^{1}}(G)$. It is also well-known (see Knudson's book [30] or Jardine's article [28]) that this rigidity property for the space $B G$ itself implies directly Friedlander's conjecture. However, though there is so far no known method to prove it for $B G$ directly, in contrast, we are able to prove it for $B \operatorname{Sing} \mathbb{A}^{1}(G)$. The latter space is a bit more flexible than $B G$, it should be thought of as the +-construction in the $\mathbb{A}^{1}$-homotopy sense, and can thus be reached by standard method of algebraic topology, in contrast to $B G$.

To prove the rigidity property at $\ell$ for $B \operatorname{Sing}_{\bullet}^{\mathbb{A}^{1}}(G)$ we will use the following Theorem which we prove in Section 3: 
THEOREM 10. Let $\mathcal{X}$ be a pointed simply-connected $\mathbb{A}^{1}$-local space over the base field $k$, $\ell$ be a prime different from char $(k)$. If the $\mathbb{A}^{1}$-chain complexes $C_{*}^{\mathbb{A}^{1}}\left(\mathcal{X}^{n} ; \mathbb{Z} / \ell\right), n \geq 1$, have the rigidity property at $\ell$, then the space $\mathcal{X}$ satisfies the rigidity property at $\ell$.

To prove the Theorem 10, we implement first in Section 3.1 the $\mathbb{A}^{1}$-lower central series, which is constructed by analogy with $[\mathbf{8}, \mathbf{9}]$ from the classical lower-central series filtration of the loop space. This gives a canonical expression of the pointed simply connected $\mathbb{A}^{1}$-local space $\mathcal{X}$ as the homotopy inverse limit of a tower of simply connected pointed spaces

$$
\cdots \rightarrow W_{s}(\mathcal{X}) \rightarrow W_{s-1}(\mathcal{X}) \rightarrow \cdots \rightarrow W_{1}(\mathcal{X}) \rightarrow *
$$

in which the homotopy fiber of $W_{s}(\mathcal{X}) \rightarrow W_{s-1}(\mathcal{X})$ is the generalized Eilenberg-MacLane space $B\left(L_{s-1}\left(K C_{*}(\mathcal{X})[-1]\right)\right)$, whose loop space is the free Lie algebra functor $L_{s-1}$ in weight $s-1$ applied to the loop space of the Eilenberg-MacLane space $K C_{*}(\mathcal{X})$ associated to the chain complex $C_{*}(\mathcal{X})$ of $\mathcal{X}$. Using $[\mathbf{8}]$ and the fundamental connectivity results from $[\mathbf{4 1}]$, we see that

$$
L_{s-1}\left(K C_{*}(\mathcal{X})[-1]\right)
$$

gets highly and highly $\mathbb{A}^{1}$-connected as $s$ increases.

The proof of Theorem 10 follows from this in Section 3.2 through a devissage of the total $\mathbb{A}^{1}$-derived functor of the free Lie algebra functors $L_{s}$, that is to say of $\mathbb{A}^{1}$-localization of spaces of the form $L_{s-1}\left(K C_{*}\right)$, for $C_{*}$ a complex of abelian sheaves (see $[\mathbf{1 1}, \mathbf{1 0}]$ for the notion of derived functors of non additive functors in the classical case).

We prove that the $\mathbb{A}^{1}$-chain complexes $C_{*}^{\mathbb{A}^{1}}\left(\left(B \operatorname{Sing}_{\bullet}^{\mathbb{A}^{1}}(G)\right)^{n} ; \mathbb{Z} / \ell\right), n \geq 1$, have the rigidity property at $\ell$ in Sections 4 and 5 . Using general simple facts on the $\mathbb{A}^{1}$-chain complexes, we show in Section 4 that the Bruhat decomposition for $G$ reduces the rigidity property at $\ell$ for the space $B \operatorname{Sing} \mathbb{A}^{\mathbb{A}^{1}}(G)$ to the rigidity property of the $\mathbb{A}^{1}$-chain complexes $C_{*}^{\mathbb{A}^{1}}\left(\left(\mathbb{G}_{m}\right)^{\wedge n}\right)$ of smash-products of $\mathbb{G}_{m}$ 's. This finishes the first step of our proof.

The rigidity property of the $\mathbb{A}^{1}$-chain complexes $C_{*}^{\mathbb{A}^{1}}\left(\left(\mathbb{G}_{m}\right)^{\wedge n}\right)$ is proven in section 5. To do this we embed the free sheaf of abelian groups

$$
\mathcal{Z}(n):=\mathbb{Z}\left(\left(\mathbb{G}_{m}\right)^{\wedge n}\right)
$$

on the $n$-th smash-power $\left(\mathbb{G}_{m}\right)^{\wedge n}$ of $\mathbb{G}_{m}$ into an explicit sheaf $\mathcal{Z}_{t r}(n)$ called the transfer completion of $\mathcal{Z}_{n}$, such that by construction, for any finite field extension $K \subset L$ with a generator $x \in L$, there is a canonical transfer morphism

$$
\operatorname{Tr}_{K}^{L}: \mathcal{Z}(n)(L) \rightarrow \mathcal{Z}_{t r}(n)(K)
$$

This sheaf $\mathcal{Z}_{t r}(n)$ must be thought of as the sheaf of finite correspondences to $\mathbb{A}^{1}$ with coefficients in $\mathcal{Z}(n)$, in the spirit of $[67]$.

We then prove in a very explicit way that the monomorphism

$$
\mathcal{Z}(n) \subset \mathcal{Z}_{t r}(n)
$$


is an $\mathbb{A}^{1}$-quasi-isomorphism, that is to say that the $\mathbb{A}^{1}$-localization functor $L_{\mathbb{A}^{1}}^{a b}(-)$ for chain complexes induces a quasi-isomorphism of chain complexes of abelian groups on $\mathrm{Sm}_{k}$

$$
C_{*}^{\mathbb{A}^{1}}\left(\left(\mathbb{G}_{m}\right)^{\wedge n}\right)=L_{\mathbb{A}^{1}}^{a b}(\mathcal{Z}(n)) \cong L_{\mathbb{A}^{1}}^{a b}\left(\mathcal{Z}_{t r}(n)\right)
$$

Some general fact on $\mathbb{A}^{1}$-localization and base change imply that the above transfers morphisms from $\mathcal{Z}(n)(L)$ to $\mathcal{Z}_{t r}(n)(K)$ induce then actual transfers morphisms on the homology sheaves of the chain complex $C_{*}^{\mathbb{A}^{1}}\left(\left(\mathbb{G}_{m}\right)^{\wedge n}\right)$.

The $\mathbb{A}^{1}$-homology sheaves of the $\mathbb{A}^{1}$-localizations are known $[\mathbf{3 8}, \mathbf{4 1}]$ to be strictly $\mathbb{A}^{1}$-invariant sheaves. A strictly $\mathbb{A}^{1}$-invariant sheaf $M$ is a sheaf of abelian groups on $S m_{k}$ such that for any $X \in S m_{k}$ the map

$$
H^{*}(X ; M) \rightarrow H^{*}\left(\mathbb{A}^{1} \times X ; M\right)
$$

is an isomorphism. Here the cohomology is computed in the Nisnevich topology. These type of sheaves form an abelian category, and the structure of transfers on those that we obtained is a new one, which we call strictly $\mathbb{A}^{1}$-invariants sheaves with generalized transfers, see Section 5.2 for a precise definition. To be slighlty more precise, this structure involves a structure of sheaf of modules over the sheaf $\underline{\mathbf{G W}}=\underline{\mathbf{K}}_{0}^{M W}$ of unramified GrothendieckWitt rings of symmetric non-degenerate bilinear forms constructed in [41] (it is called there the Milnor-Witt $K$-theory ring in weight 0 ), which is compatible in a tricky sense with the transfers. The structure of homotopy invariant sheaves with transfers defined by Voevodsky [67] is exactly the particular case where the $\mathbf{G W}$-module structure is trivial, that is to say factors through the rank homomorphism $\mathbf{G W} \rightarrow \mathbb{Z}$.

We conclude the proof of the rigidity property for the $\mathbb{A}^{1}$-chain complexes $C_{*}^{\mathbb{A}^{1}}\left(\left(\mathbb{G}_{m}\right)^{\wedge n}\right)$ by observing first that for $\ell$ odd, when -1 is a square in the base field, the $\mathbf{G W}$-module structure on the $\bmod \ell$ homology sheaves has to be trivial, thus giving homotopy invariant sheaves (of $\mathbb{Z} / \ell$-vector spaces) with transfers in the classical sense of Voevodsky. These sheaves have the rigidity property by $[\mathbf{6 3}]$. Theorem 3 for odd $\ell$ follows from this.

To prove the rigidity property at the prime $\ell=2$ requires a bit more work. Roughly speaking we observe that for a strictly $\mathbb{A}^{1}$-invariant sheaf $M$ with generalized transfers, one may define a filtration

$$
\cdots \subset \mathcal{I}^{n} M \subset \cdots \subset \mathcal{I} M \subset M
$$

by strictly $\mathbb{A}^{1}$-invariant sheaves stable under the transfers and the $\mathbf{G W}$ module structures which is directly induced by the filtration of $\mathbf{G W}$ by the powers of the fundamental ideal $\underline{\mathbf{I}} \subset \mathbf{G W}$. The successive subquotients $\mathcal{I}^{n} M / \mathcal{I}^{n+1} M$ are thus homotopy invariant sheaves with transfers in the sense of Voevodsky and thus satisfy the rigidity property at 2 as well.

To conclude in the case $\ell=2$ using this filtration, we observe that for a strict henselization $A$ of a (geometric) point in a smooth $k$-scheme with fraction field $F$ one has $\mathcal{I}^{n} M(F)=0$ for $n \geq 2$. One thus concludes from the rigidity property of $\mathcal{I}^{n} M / \mathcal{I}^{n+1} M$ for $n=0$ and 1 . 
We also observe that just the existence of the subsheaf $\mathcal{I} M \subset M$ is enough to prove the rigidity property at 2 in the original sense of Suslin, that the mod 2 homology $H_{*}\left(B \operatorname{Sing}_{\bullet}^{\mathbb{A}^{1}}(G)(F) ; \mathbb{Z} / 2\right)$ doesn't depend on the separably closed field $F$. In finite characteristic this suffices to prove Theorem 3 by the results of $[\mathbf{1 4}]$.

REMARK 11. Using the affirmation by Voevodsky of the Milnor conjectures, which implies (see [2, Lemma 2.1]) that for a field $F$ of finite type over its prime field, $I^{n+1}(F)=2 I^{n}(F)$ for $n$ large enough, we get a much stronger rigidity property, which holds for any henselization of a point of a smooth $k$-scheme.

The property that we actually can prove for the space $B \operatorname{Sing} \mathbb{A}^{\mathbb{A}^{1}}(G)$ is indeed much stronger that just to imply the weak Friedlander conjecture. It implies also in case -1 is a square in $k$ that the canonical morphism

$$
B \operatorname{Sing} \mathbb{A}_{\bullet}^{\mathbb{A}^{1}}(G) \rightarrow\left(B \operatorname{Sing} \mathbb{A}_{\bullet}^{\mathbb{A}^{1}}(G)\right)_{\mu}^{\wedge}
$$

from the space to its motivic completion, obtained either by the method of Sullivan [58] or directly as the Bousfield-Kan Tot construction [5] applied to the cosimplicial space obtained by using the pair of adjoint functors "Voevodsky motive" and "motivic Eilenberg-MacLane space", is a weakequivalence when evaluated at fields (or Henselian local rings). We will come back to this point in a sequel to this work. One says then that $\operatorname{BSing} \mathbb{A}^{1}(G)$ is motivic complete.

To emphasize our method, let us mention the following result which is a generalization of Theorem 3 (obtained by taking for $k$ an algebraic closure of a prime field and $\left.\mathcal{X}=B \operatorname{Sing} \mathbb{A}^{\mathbb{A}^{1}}(G)\right)$ :

TheOREM 12. Assume $k$ is an algebraically closed field, $\ell$ is a prime number different from char $(k)$ and let $\mathcal{X}$ be a pointed $\mathbb{A}^{1}$-local simply connected space over $k$. If the $\mathbb{A}^{1}$-chain complex $x^{1}$ of $\mathcal{X}$ is of mixed Tate type $e^{2}$, then $\mathcal{X}$ is motivic complete, and in particular has the rigidity property at $\ell$ (see Section 2.1).

In particular, for any extension $k \subset F$ with $F$ separably closed, the morphism of simplicial sets $\mathcal{X}(k) \rightarrow \mathcal{X}(F)$ induces an isomorphism

$$
H^{*}(\mathcal{X}(F) ; \mathbb{Z} / \ell) \cong H^{*}(\mathcal{X}(k) ; \mathbb{Z} / \ell)
$$

and moreover the natural ring homomorphism

$$
H_{e t}^{*}(\mathcal{X} ; \mathbb{Z} / \ell) \rightarrow H^{*}(\mathcal{X}(F) ; \mathbb{Z} / \ell)
$$

from the $\mathbb{Z} / \ell$-étale cohomology of the space $\mathcal{X}$ to the mod $\ell$ cohomology of $\mathcal{X}(F)$ is an isomorphism.

\footnotetext{
$1_{\text {see }[39][41]}$

2 see Section 4.1 , this basically means that it is build out of $C_{*}^{\mathbb{A}^{1}}\left(\mathbb{G}_{m}^{\wedge n}\right)$ 's
} 
REMARK 13. A further consequence of this result is that, when $k$ is algebraically closed and $G$ is a semi-simple $k$-group, the morphism of pro- $\ell$ simplicial sets

$$
\left(B \operatorname{Sing} \mathbb{A}^{1}(G)(k)\right)_{\ell}^{\wedge} \rightarrow\left(B \operatorname{Sing} \mathbb{A}_{\bullet}^{\mathbb{A}^{1}}(G)\right)_{\ell, e t}=(B G)_{\ell, e t}^{\wedge}
$$

and also

$$
\left(\operatorname{Sing}_{\bullet}^{\mathbb{A}^{1}}(G)(k)\right)_{\ell} \rightarrow\left(\operatorname{Sing}_{\bullet}^{\mathbb{A}^{1}}(G) \hat{r}_{\ell, e t}=(G)_{\ell, e t}^{\hat{C}_{\ell}}\right.
$$

obtained by applying the pro- $\ell$ étale homotopy type functor (see for instance $[\mathbf{3}, \mathbf{1 3}])$ to the inclusions of spaces $\operatorname{BSing}_{\mathbf{A}^{1}}(G)(k) \subset B \operatorname{Sing} \mathbb{A}^{1}(G)$ or $\operatorname{Sing} \mathbb{A}_{\bullet}^{\mathbb{A}^{1}}(G)(k) \subset \operatorname{Sing} g_{\bullet}^{\mathbb{A}^{1}}(G)$ is an equivalence. Our technic may be used to extend these results to the Suslin-Voevodsky construction on other smooth $k$-schemes. For instance one gets a pro- $\ell$ equivalence of the form

$$
\operatorname{Sing}_{\bullet}^{\mathbb{A}^{1}}\left(\mathbb{P}^{n}\right)(k)_{\ell}^{\wedge} \cong\left(\mathbb{P}^{n}\right)_{\ell, e t}^{\wedge}
$$

by applying the pro- $\ell$ étale homotopy type functor to the inclusion of spaces $\left.\operatorname{Sing}_{\bullet}^{\mathbb{A}^{1}}\left(\mathbb{P}^{n}\right)(k)\right) \subset \operatorname{Sing}_{\bullet}^{\mathbb{A}^{1}}\left(\mathbb{P}^{n}\right)$. More generally one may derive such equivalences for the Suslin-Voevodsky construction $\operatorname{Sing}_{\bullet}^{\mathbb{A}^{1}}(X)$ on various homogeneous varieties of the form $X:=G / H$, where $H$ is a $k$-subgroup of $G$.

This proves that under the previous assumptions, the "very naive" $\bmod$ $\ell$ homology of the simplicial set $\operatorname{Sing}_{\bullet}^{\mathbb{A}^{1}}(X)(k)$ coincides with the $\bmod \ell$ Suslin homology of $X$ as it follows from [63]. This result is also new.

Conventions. For a field $F$, we let $S m_{F}$ denote the category of finite type smooth $F$-schemes.

In the sequel, $k$ denotes a fixed perfect field, $G$ a fixed split semi-simple $k$-group, and $\ell$ denotes a prime number different from $\operatorname{char}(k)$. We let $\mathcal{F}_{k}$ denote the category of fields extensions of $k$ which are of finite transcendence degree over $k$.

If not otherwise stated, by sheaf we always mean a sheaf in the Nisnevich topology on $S m_{k}$ and by a space we mean a simplicial sheaf of sets in the Nisnevich topology on $S m_{k}$.

We refer the reader to $[\mathbf{4 4 , 4 1 ]}$ for the basic notions concerning the simplicial and $\mathbb{A}^{1}$-homotopy theory of sheaves, the notion of chain complexes and their corresponding Eilenberg-MacLane spaces, and the notions of $\mathbb{A}^{1}$ homotopy sheaves, $\mathbb{A}^{1}$-homology sheaves and $\mathbb{A}^{1}$-chain complexes, which we will use freely.

We will finally assume the reader is acquainted with the notion of essentially smooth $k$-schemes, these are for us noetherian $k$-schemes obtained as inverse limit of a system of smooth $k$-schemes in which the transitions morphisms are étale and affine. For instance the spectrum of the local ring $\mathcal{O}_{X, x}$ of a smooth $k$-scheme $X$ at a point $x$. Or its henselization $\mathcal{O}_{X, x}^{h}$. For such an essentially smooth $k$-scheme $U=\lim _{\alpha} U_{\alpha}$, and for $\mathcal{X}$ a space, we will denote by $\mathcal{X}(U)$ the simplicial set obtained as the filtering colimit of the $\mathcal{X}\left(U_{\alpha}\right)$ 's. For instance, if $F \in \mathcal{F}_{k}, \operatorname{Spec}(F)$ is essentially smooth over $k$, and $\mathcal{X}(F)$ is the stalk of $\mathcal{X}$ at $F$. 
Acknowledgement. This work is part of a systematic study of the $\mathbb{A}^{1}$-homotopy type of classifying spaces of algebraic groups started years ago, aiming at understanding the Serre conjectures in Galois cohomology from the point of view of $\mathbb{A}^{1}$-homotopy theory. I am grateful to Aravind Asok for mentioning to me at some point the Friedlander conjecture. I want to thank Vladimir Voevodsky for his interest during my stay at the Institute for Advanced Studies in 2010. I also want to specially thank Mike Hopkins for his interest in this work, for his support and for some helpful discussions.

The final idea which led to a very important simplification of a previous construction, related to the construction of some transfers morphisms on the $\mathbb{A}^{1}$-chain complexes of tori, is born at the I.A.S. in the spring 2010. I am very gateful to the I.A.S. for his hospitality during this period January-May 2010, which allowed me to improved considerably the present work in many aspects.

\section{The $\mathbb{A}^{1}$-local space $B \operatorname{Sing} \mathbb{A}_{\bullet}^{1}(G)$}

1.1. The Suslin-Voevodsky construction. For $\mathcal{M}:\left(S m_{k}\right)^{o p} \rightarrow$ Set a presheaf of sets on the category of smooth $k$-schemes, we may consider the simplicial presheaf of sets on $S m_{k}$ defined by

$$
U \mapsto \mathcal{M}\left(\Delta^{\bullet} \times U\right)
$$

where $n \mapsto \Delta_{k}^{n}=\Delta^{n}:=\operatorname{Spec}\left(k\left[T_{0}, \ldots, T_{n}\right] / \sum T_{i}=1\right)$ is the standard algebraic cosimplicial simplex over $F[63]$. If $\mathcal{M}$ is a sheaf (in the Nisnevich topology) on $S m_{k}$, this simplicial presheaf is a simplicial sheaf of sets, which we denote by $\operatorname{Sing}_{\bullet}^{\mathbb{A}^{1}}(\mathcal{M})$. We will call this construction the Suslin-Voevodsky construction on $\mathcal{M}$.

We will be interested in the Suslin-Voevodsky construction $\operatorname{Sing}_{\mathbf{A}^{1}}(G)$ on $G$ itself. It is a simplicial sheaf of groups on $S m_{k}$, in fact a sheaf in the étale topology as well, but at this point we will ignore it. We may consider its simplicial classifying space $B \operatorname{Sing} \mathbb{A}^{\mathbb{A}^{1}}(G)$ defined by the usual formulas (see for instance [44, page 128]). It is a simplicial sheaf of sets on $S m_{k}$ and we have a canonical inclusion of simplicial sheaves of sets (coming from the inclusion $G \subset \operatorname{Sing}_{\bullet}^{\mathbb{A}^{1}}(G)$ as the sheaf of 0-vertices)

$$
B G \rightarrow B \operatorname{Sing}_{\bullet}^{\mathbb{A}^{1}}(G)
$$

Let $\ell$ be a prime number different from $\operatorname{char}(F)$ and let $M$ be a locally constant sheaf of $\mathbb{Z} / \ell$-torsion abelian groups on $S m_{F}$; the classical $\mathbb{A}^{1}$-invariance property of $H_{e t}^{*}(-; M)[\mathbf{2 3}]$ and the argument of the proof of $[\mathbf{4 4}$, Corollary 3.8 p. 89] imply:

THEOREM 1.1. The morphism $B G \rightarrow$ BSing $_{\bullet}^{\mathbb{A}^{1}}(G)$ induces an isomorphism

$$
H_{e t}^{*}\left(B \operatorname{Sing}_{\bullet}^{\mathbb{A}^{1}}(G) ; M\right) \cong H_{e t}^{*}(B G ; M)
$$


Using the previous results, Theorem 3 for $G$ and $F$ is now clearly a consequence of the following result, by taking for $k$ the algebraic closure (in $F$ ) of the prime field:

TheOrem 1.2. Assume $k$ is algebraically closed. Let $F$ be a separably closed field extension of $k$ and let $G$ be a semi-simple $k$-group. The natural ring homomorphism

$$
H_{e t}^{*}\left(B \operatorname{Sing}_{\bullet}^{\mathbb{A}^{1}}(G) ; \mathbb{Z} / \ell\right) \rightarrow H^{*}\left(B \operatorname{Sing}_{\bullet}^{\mathbb{A}^{1}}(G)(F) ; \mathbb{Z} / \ell\right)
$$

is an isomorphism.

This is our starting point. We will refer to this result as the "weak Friedlander conjecture". The space $B \operatorname{Sing}_{\mathbf{A}^{1}}{ }^{1}(G)$ is somehow much more flexible than the simplicial classifying space $B G$ as it will appear in the next sections. For instance for $G$ simply connected (as an algebraic $k$-group) $B \operatorname{Sing} \mathbb{A}^{\mathbb{A}^{1}}(G)$ is a $\left(\mathbb{A}^{1}\right.$-) simply connected space by Theorem 1.5 below, though in contrast $B G$ has for only non trivial simplicial homotopy sheaf its $\pi_{1}$ which is $G$ itself! The space $B \operatorname{Sing} \mathbb{A}^{1}(G)$ may be thought in some sense as the + -construction on $B G$. The morphism

$$
B G \rightarrow B \operatorname{Sing}_{\bullet}^{\mathbb{A}^{1}}(G)
$$

induces an isomorphism on $\mathbb{A}^{1}$-homology (see below) and $B \operatorname{Sing} \mathbb{A}^{1}(G)$ is $\mathbb{A}^{1}$-simply connected. Its homotopy sheaves in contrast to that of $B G$ are unknown (expect the first one by the Hurewicz Theorem), but these are much more flexible objects!

1.2. The $\mathbb{A}^{1}$-local space $\operatorname{Sing}_{\bullet}^{\mathbb{A}^{1}}(G)$ and its classifying space. In [41] we proved that $\operatorname{Sing}_{\mathbb{A}^{1}}\left(S L_{n}\right)$, for $n \geq 3$, is $\mathbb{A}^{1}$-local. Recall from [44] that a space $\mathcal{X}$ is $\mathbb{A}^{1}$-local if and only if the morphism to its $\mathbb{A}^{1}$-localization

$$
\mathcal{X} \rightarrow L_{\mathbb{A}^{1}}(\mathcal{X})
$$

is a simplicial weak equivalence. We proved in fact a much stronger property of $\operatorname{Sing}_{\bullet}^{\mathbb{A}^{1}}\left(S L_{n}\right)$ : it has the affine $\mathbb{A}^{1}$-Brown-Gersten property in the Nisnevich topology. This result has been generalized to any semi-simple $k$-group $G$ by Wendt [69], except for the type $S L_{2}$, which has recently been settled by L.-F. Moser. Thus:

THEOREM 1.3. ([41] for $S L_{n}, n \geq 3$, Wendt [69] for type different of $S L_{2}$, Moser [45] for $\left.S L_{n}, n \geq 2\right)$ The Suslin-Voevodsky construction Sing $\mathbb{A}^{1}(G)$ of a split semi-simple $k$-group is $\mathbb{A}^{1}$-local.

Remark 1.4. 1) Let $X \in S m_{k}$. We will say that $X$ is chain $\mathbb{A}^{1}$-local if the Suslin-Voevodsky construction $\operatorname{Sing}_{\bullet}^{\mathbb{A}^{1}}(X)$ on $X$ is $\mathbb{A}^{1}$-local. In fact I do not know any example of $X$ which is not $\mathbb{A}^{1}$-chain local.

2) Let $M$ be a sheaf of abelian groups with transfers in the sense of Voevodsky [67]. Then one of the consequences of the results of Voevodsky on sheaves with transfers $[\mathbf{6 7}][\mathbf{6 8}]$ is that the spaces $\operatorname{Sing}_{\bullet}^{\mathbb{A}^{1}}(M), B \operatorname{Sing} \mathbb{A}_{\bullet}^{\mathbb{A}^{1}}(M)$, 
$K\left(\operatorname{Sing}_{\bullet}^{\mathbb{A}^{1}}(M), n\right)$, etc $\ldots$ are $\mathbb{A}^{1}$-local. The difficulty in proving Theorem 1.3 is of course that the sheaf of groups $G$ is not abelian and has no reasonable structure of transfers.

The following result is a non-trivial consequence of Theorem 1.3:

TheOREM 1.5. 1) The space BSing $\mathbb{A}^{1}(G)$ is $\mathbb{A}^{1}$-local.

2) Assume furthermore that $G$ is simply connected (and split semisimple) as an algebraic k-group. Then the $\mathbb{A}^{1}$-local space $B \operatorname{Sing}_{\mathbf{A}^{1}}(G)$ is $\mathbb{A}^{1}$-simply connected.

Proof. The first statement follows from Theorem 1.3, [41, Thm 3.46] and the Theorem A.2. Now if $G$ is split semi-simple simply connected, $G$ is $\mathbb{A}^{1}$-connected by Theorem A.2 again, and thus $B G$ is simply $\mathbb{A}^{1}$-connected.

REMARK 1.6. This is the only place where we use the assumption char $(k) \neq 2$. As so far Theorem 1.3 only holds in characteristic not 2 .

\section{The Friedlander conjecture and the rigidity property}

2.1. The rigidity properties at $\ell$. We now introduce the rigidity property at $\ell$ concerning a space. A discussion of rigidity properties and various implications towards the Friedlander-Milnor conjecture may be found in $[\mathbf{3 0}, \S 5.3]$. We consider 3 notions, inspired by the rigidity Theorem of [63] and the approach of $[\mathbf{2 8}]$, though in fact we are able to prove the strongest possible one.

Below we show that if the space $B \operatorname{Sing}_{\bullet}^{\mathbb{A}^{1}}(G)$ has the rigidity property then the weak Friedlander conjecture holds for $G$ and any separably closed field extension $F$ of $k$.

Definition 2.1. Let $\mathcal{X}$ be a space.

1) We will say that $\mathcal{X}$ has the weak rigidity property at $\ell$ if for any extension $k \subset F$ with $F$ a separably closed field, the natural map of simplicial sets $\mathcal{X}\left(k_{s}\right) \rightarrow \mathcal{X}(F)$ induces an isomorphism in $\bmod \ell$ homology:

$$
H_{*}\left(\mathcal{X}\left(k_{s}\right) ; \mathbb{Z} / \ell\right) \cong H_{*}(\mathcal{X}(F) ; \mathbb{Z} / \ell)
$$

(Here $k_{s} \subset F$ means the separable closure of $k$ in $F$, which is of course separably closed itself.) When no confusion can occur on $\ell$ which is fixed, we will just say " $\mathcal{X}$ has the weak rigidity property" instead of " $\mathcal{X}$ has the weak rigidity property at $\ell$ ".

2) We will say that $\mathcal{X}$ has the rigidity property at $\ell$ if for each strict henselization $\mathcal{O}_{X, x}^{s h}$ of the local ring of a point $x \in X \in S m_{k}$ with separably closed residue field $\kappa(x)_{s}$, the natural map of simplicial sets $\mathcal{X}\left(\mathcal{O}_{X, x}^{s h}\right) \rightarrow \mathcal{X}\left(\kappa(x)_{s}\right)$ induced by restriction to the closed point induces an isomorphism in $\bmod \ell$ homology:

$$
H_{*}\left(\mathcal{X}\left(\mathcal{O}_{X, x}^{s h}\right) ; \mathbb{Z} / \ell\right) \cong H_{*}\left(\mathcal{X}\left(\kappa(x)_{s}\right) ; \mathbb{Z} / \ell\right)
$$


When no confusion can occur on $\ell$, we will just say " $\mathcal{X}$ has the rigidity property" instead of " $\mathcal{X}$ has the rigidity property at $\ell$ ".

3) We will say that $\mathcal{X}$ has the strong rigidity property at $\ell$ if for each henselization $\mathcal{O}_{X, x}^{h}$ of the local ring of a point $x \in X \in S m_{k}$ with residue field $\kappa(x)$, the natural map of simplicial sets $\mathcal{X}\left(\mathcal{O}_{X, x}^{h}\right) \rightarrow$ $\mathcal{X}(\kappa(x))$ induced by restriction to the closed point induces an isomorphism in mod $\ell$ homology:

$$
H_{*}\left(\mathcal{X}\left(\mathcal{O}_{X, x}^{h}\right) ; \mathbb{Z} / \ell\right) \cong H_{*}(\mathcal{X}(\kappa(x)) ; \mathbb{Z} / \ell)
$$

When no confusion can occur on $\ell$, we will just say " $\mathcal{X}$ has the strong rigidity property" instead of " $\mathcal{X}$ has the strong rigidity property at $\ell "$.

EXAmPle 2.2. The simplicial classifying space $B \mathbb{G}_{m}$ of the multiplicative group $\mathbb{G}_{m}$ has the strong rigidity property at $\ell$. Indeed to check this we have to prove that for each henselization $A:=\mathcal{O}_{X, x}^{h}$ of a point $x \in X \in S m_{k}$ with residue field $\kappa=\kappa(x)$ the morphism "restriction to the closed point"

$$
B\left(A^{\times}\right) \rightarrow B\left(\kappa^{\times}\right)
$$

induces an isomorphism on mod $\ell$-homology for $\ell$ different from the characteristic of $\kappa$. It suffices to check that the kernel $K$ of the surjective group homomorphism $A^{\times} \rightarrow \kappa^{\times}$has trivial mod $\ell$-homology; for this it suffices to observe that $K$ is uniquely $\ell$-divisible, which follows directly from the classical definition of an henselian local ring.

Observe that $\mathbb{G}_{m}$ being $\mathbb{A}^{1}$-rigid, $B \mathbb{G}_{m}=B \operatorname{Sing} \mathbb{A}^{1}\left(\mathbb{G}_{m}\right)$.

Observe however that the multiplicative group $\mathbb{G}_{m}$ itself, considered as a space (placed in simplicial degree 0 ) has not the rigidity property at $\ell$.

REMARK 2.3. 1) The main result of this work is to prove that more generally, the space $B \operatorname{Sing} \mathbb{A}_{\bullet}^{1}(G)$ has the strong rigidity property at $\ell$, for $G$ split semi-simple (see Theorem 2.17).

2) It doesn't follow that the space $B G$ itself has the rigidity property at $\ell$. It sounds reasonable to believe this anyway. This would follow from our main result (Theorem 2.17) and from the following property of "homotopy invariance for group cohomology on henselian rings": let $\mathcal{O}_{X, x}^{h}$ be the Henselization of a point $x \in X \in S m_{k}$ then the morphism

$$
H_{*}\left(B G\left(\mathcal{O}_{X, x}^{h}\right) ; \mathbb{Z} / \ell\right) \rightarrow H_{*}\left(B G\left(\mathcal{O}_{X, x}^{h}\left[T_{1}, \ldots, T_{n}\right]\right) ; \mathbb{Z} / \ell\right)
$$

is an isomorphism for each $n$.

The following Lemma will be useful:

Lemma 2.4. Assume that $\mathcal{F} \rightarrow \mathcal{E} \rightarrow \mathcal{B}$ is a simplicial fibration between fibrant pointed spaces, with $\mathcal{B}$ connected. Assume that for any $x \in X \in S m_{k}$ the action of $\pi_{1}\left(\mathcal{B}\left(\mathcal{O}_{X, x}^{h}\right)\right)$ on the $\bmod \ell$-homology of $\mathcal{F}\left(\left(\mathcal{O}_{X, x}^{h}\right)\right)$ is trivial (for instance if $\mathcal{B}$ is simply connected or if the fibration is up to weak equivalence 
a principal fibration with group $\mathcal{G} \cong \mathcal{F}$ connected). Then if two of $\mathcal{B}, \mathcal{E}$ or $\mathcal{F}$ have the rigidity property of type $i \in\{1,2,3\}$, then so has the third.

PROOF. Indeed, let us do the case of type 3, the strong rigidity property. For each point $x \in X \in S m_{k}$ with residue field $\kappa(x)$ we may consider the two Serre homology spectral sequences with $\bmod \ell$ coefficients of the two fibrations sequences of simplicial sets $\mathcal{F}\left(\mathcal{O}_{X, x}^{h}\right) \rightarrow \mathcal{E}\left(\mathcal{O}_{X, x}^{h}\right) \rightarrow \mathcal{B}\left(\mathcal{O}_{X, x}^{h}\right)$ and $\mathcal{F}(\kappa(x)) \rightarrow \mathcal{E}(\kappa(x)) \rightarrow \mathcal{B}(\kappa(x))$. From the assumptions, both $E^{2}$ terms are of the form $H_{*}(\mathcal{B} ; \mathbb{Z} / \ell) \otimes H_{*}(\mathcal{F} ; \mathbb{Z} / \ell)$ (meaning that the local coefficients systems are constant). Using the naturality of the Serre spectral sequence, the rest of the argument is classical.

REMARK 2.5. For instance, assume that $\mathcal{F} \rightarrow \mathcal{E} \rightarrow \mathcal{B}$ is a simplicial fibration between fibrant pointed spaces with $\mathcal{B}$ simply connected and $\mathcal{E}$ weakly contractible. In other words $\mathcal{F}$ has the weak simplicial homotopy type of the simplicial loop space $\Omega(\mathcal{B})$. Then the previous Lemma tells us that the following properties are equivalent:

(1) $\mathcal{B}$ has the rigidity property of type $i$,

(2) $\Omega(\mathcal{B})$ has the rigidity property of type $i$.

Example 2.2 shows that in general the result (and thus Lemma 2.4) doesn't hold without assumptions on the $\pi_{1}$ of $\mathcal{B}$.

Let us mention an application without proof:

LEMMA 2.6. For any split semi-simple $k$-group $G$ with universal covering $\tilde{G} \rightarrow G$ (in the sense of algebraic group theory), if $B \operatorname{Sing}_{\bullet}^{\mathbb{A}^{1}}(\tilde{G})$ has the rigidity property, then $B$ Sing $\mathbb{A}^{1}(G)$ has the rigidity property.

In what follows we give a not very surprising characterization of pointed simply connected space $\mathcal{X}$ which have the rigidity property in term of their simplicial homotopy sheaves. We leave to the reader to state and prove the analogue statement involving the weak (resp. strong) rigidity property. Recall that for $M$ an abelian group we let respectively ${ }_{[\ell]} M$ and $M / \ell$ be the kernel and the cokernel of the homomorphism "multiplication by $\ell$ ": $M \stackrel{\ell}{\longrightarrow} M$.

TheOREM 2.7. Let $\mathcal{X}$ be a pointed simply connected space. The following conditions are equivalent:

(i) $\mathcal{X}$ has the rigidity property;

(ii) for each integer $n \geq 2$ and for each henselization $\mathcal{O}_{X, x}^{h}$ of the local ring of a point $x \in X \in S m_{k}$ with residue field $\kappa(x)$, the kernel of the natural epimorphism $\pi_{n}(\mathcal{X})\left(\mathcal{O}_{X, x}^{h}\right) \rightarrow \pi_{n}(\mathcal{X})(\kappa(x))$ is uniquely $\ell$-divisible.

(iii) for each integer $n \geq 2$ and for each henselization $\mathcal{O}_{X, x}^{h}$ of the local ring of a point $x \in X \in S m_{k}$ with residue field $\kappa(x)$, the two homorphisms ${ }_{[\ell]} \pi_{n}(\mathcal{X})\left(\mathcal{O}_{X, x}^{h}\right) \rightarrow{ }_{[\ell]} \pi_{n}(\mathcal{X})(\kappa(x))$ and $\pi_{n}(\mathcal{X})\left(\mathcal{O}_{X, x}^{h}\right) / \ell \rightarrow$ $\pi_{n}(\mathcal{X})(\kappa(x)) / \ell$ are isomorphisms. 
Proof. The equivalence $(i i) \Leftrightarrow(i i i)$ is clear. The implication $(i i) \Rightarrow(i)$ is proven using the Postnikov tower $\left\{P^{(n)}(\mathcal{X})\right\}_{n}$ of $\mathcal{X}$ and follows by induction on $n$ from the fact that for each $n$ the map $K\left(\pi_{n}(\mathcal{X})\left(\mathcal{O}_{X, x}^{h}\right), n\right) \rightarrow$ $K\left(\pi_{n}(\mathcal{X})(\kappa(x)), n\right)$ induces by assumption an isomorphism on $\bmod \ell$ homology. To prove the converse implication $(i) \Rightarrow(i i)$ one uses induction on $r$ and the Lemma 2.8 below.

LEMMA 2.8. Let $f: K \rightarrow L$ be a morphism of pointed simply connected simplicial sets which admits a section in the pointed homotopy category, let $\ell$ be a prime number and let $r \geq 1$ be a natural number. The following conditions are equivalent:

(i) the morphism $f$ induces an isomorphism on $H_{*}(-; \mathbb{Z} / \ell)$ for $* \leq r$;

(ii) let $\Gamma$ be the homotopy fiber of $f$; then the homology $H_{*}(\Gamma ; \mathbb{Z} / \ell)$ is trivial for $* \leq r$;

(iii) the kernel of the (split) epimorphism $\pi_{i}(K) \rightarrow \pi_{i}(L)$ is uniquely $\ell$-divisible for $i \leq r-1$ and $\ell$-divisible for $i=r$. In other words ${ }_{[\ell]} \pi_{i}(K) \rightarrow{ }_{[\ell]} \pi_{i}(L)$ is an isomorphism for $i \leq r-1$ and $\pi_{i}(K) / \ell \rightarrow$ $\pi_{i}(L) / \ell$ is an isomorphism for $i \leq r$.

2.2. Reformulation of the rigidity property in term of simplicial chain complex. We let $\mathcal{A} b(k)$ denote the abelian category of sheaves of abelian groups in the Nisnevich topology on $S m_{k}$, and $\mathcal{C}_{*}(\mathcal{A} b(k))$ the category of chain complexes in $\mathcal{A} b(k)$ (with differential of degree -1$)$. Given a space $\mathcal{X}$ we let $\mathbb{Z}(\mathcal{X})$ denote the free simplicial sheaf of abelian groups generated by $\mathcal{X}$, that is to say the sheafification of the presheaf $U \mapsto \mathbb{Z}(\mathcal{X}(U))$ obtained by taking (degreewise) the free abelian group generated by the simplicial set $\mathcal{X}(U)$. This is a simplicial sheaf of abelian groups, and we may associate to it its normalized chain complex $C_{*}(\mathcal{X} ; \mathbb{Z}) \in \mathcal{C}_{*}(\mathcal{A} b(k)$ ) (see for instance $[\mathbf{9}]$ for the definition of normalized chain complex). This chain complex is called the simplicial chain complex $x^{3}$ of $\mathcal{X}$. We let $C_{*}(\mathcal{X} ; \mathbb{Z} / \ell)$ be the $\bmod \ell$ version, which means the tensor product $C_{*}(\mathcal{X} ; \mathbb{Z}) \otimes \mathbb{Z} / \ell=$ $C_{*}(\mathcal{X} ; \mathbb{Z}) / \ell$.

The $n$-th homology sheaf of $C_{*}(\mathcal{X} ; \mathbb{Z})$ (resp. $\left.C_{*}(\mathcal{X}: \mathbb{Z} / \ell)\right)$ is called the $n$-th simplicial homology sheaf of $\mathcal{X}$ with $\mathbb{Z}$ (resp. $\mathbb{Z} / \ell$ ) coefficients and is denoted by $H_{n}(\mathcal{X} ; \mathbb{Z})\left(\right.$ resp. $\left.H_{n}(\mathcal{X} ; \mathbb{Z} / \ell)\right)$.

Definition 2.9. 1) A chain complex $C_{*}$ of $\mathbb{Z} / \ell$-modules will be said to have the rigidity property (at $\ell$ ) if and only if for each henselization $\mathcal{O}_{X, x}^{h}$ of the local ring of a point $x \in X \in S m_{k}$ with residue field $\kappa(x)$, the natural morphism of chain complexes of $\mathbb{Z} / \ell$-modules

$$
C_{*}\left(\mathcal{O}_{X, x}^{h}\right) \rightarrow C_{*}(\kappa(x))
$$

is a quasi-isomorphism.

\footnotetext{
3 as opposed to the $\mathbb{A}^{1}$-chain complex which will appear later
} 
2) A chain complex $C_{*}$ of abelian groups will be said to have the rigidity property $\left(\right.$ at $\ell$ ) if and only if for each henselization $\mathcal{O}_{X, x}^{h}$ of the local ring of a point $x \in X \in S m_{k}$ with residue field $\kappa(x)$, the natural morphism of chain complexes of $\mathbb{Z} / \ell$-modules

$$
C_{*}\left(\mathcal{O}_{X, x}^{h}\right) \otimes^{L} \mathbb{Z} / \ell \rightarrow C_{*}(\kappa(x)) \otimes^{L} \mathbb{Z} / \ell
$$

is a quasi-isomorphism (in other words if $C_{*} \otimes^{L} \mathbb{Z} / \ell$ has the rigidity property).

EXAMPLE 2.10. 1) A complex of sheaves with transfers in the sense of Voevodsky $[\mathbf{6 7}]$ whith $\mathbb{A}^{1}$-invariant homology sheaves has the rigidity property at $\ell$ by $[\mathbf{6 3}]$.

2) Let $M$ be a sheaf of $\mathbb{Z} / \ell$-modules. Then the complex $M$ (placed in degree 0 ) has the rigidity property at $\ell$ if and only if for each henselization $\mathcal{O}_{X, x}^{h}$ of the local ring of a point $x \in X \in S m_{k}$ with residue field $\kappa(x)$, the natural epimorphism of $\mathbb{Z} / \ell$-modules $M\left(\mathcal{O}_{X, x}^{h}\right) \rightarrow M(\kappa(x)$ is an isomorphism.

3) Let $M$ be a sheaf of abelian groups. Then the complex $M$ (placed in degree 0 ) has the rigidity property at $\ell$ if and only if the sheaves $M / \ell$ and ${ }_{[\ell]} M$ (kernel of the multiplication by $\ell$ ) have the rigidity property. Of course as these two sheaves are sheaves of $\mathbb{Z} / \ell$-modules, we may use the characterization given in the preceding point.

Lemma 2.11. 1) Let $\mathcal{X}$ be a space. The following conditions are equivalent:

(i) the space $\mathcal{X}$ has the rigidity property at $\ell$;

(ii) the chain complex $C_{*}(\mathcal{X} ; \mathbb{Z} / \ell)$ has the rigidity property at $\ell$.

2) Let $C_{*}$ be a chain complex of sheaves of $\mathbb{Z} / \ell$-modules and let $K C_{*}$ be its corresponding Eilenberg-MacLane space. If $H_{*}\left(C_{*}\right)=0$ for $*<0$, the following conditions are equivalent:

(i) the space $K C_{*}$ has the rigidity property at $\ell$;

(ii) the chain complex $C_{*}$ has the rigidity property.

3) Let $C_{*}$ be a chain complex of sheaves of abelian groups and let $K C_{*}$ be its corresponding Eilenberg-MacLane space. If $H_{*}\left(C_{*}\right)=0$ for $* \leq 0$, the following conditions are equivalent:

(i) the space $K C_{*}$ has the rigidity property at $\ell$;

(ii) the chain complex $C_{*}$ has the rigidity property.

Proof. The point 1) is clear. The point 2) follows from the following classical fact: a morphism $A_{\bullet} \rightarrow B_{\bullet}$ between two simplicial $\mathbb{Z} / \ell$-modules is a simplicial weak equivalence if and only if it induces a isomorphism in the $\bmod \ell$ homology of the underlying simplicial sets. The third point follows easily from this, taking into account the connectivity assumption.

REMARK 2.12. Observe that one can't remove the assumption $H_{*}\left(C_{*}\right)=$ 0 for $* \leq 0$ in the last point, as for instance the chain complex $\mathbb{G}_{m}$ itself has 
the rigidity property at $\ell$ (compute $H_{*}\left(\mathbb{G}_{m} \otimes^{L} \mathbb{Z} / \ell\right)$ and check it!) but the space $\mathbb{G}_{m}$ doesn't have the rigidity property at $\ell$.

We may now prove:

TheOREM 2.13. Assume $k$ is algebraically closed and let $\mathcal{X}$ be a space which has the rigidity property at $\ell$. Then the canonical morphism of complexes of sheaves

$$
C_{*}(\mathcal{X}(k) ; \mathbb{Z} / \ell) \rightarrow C_{*}(\mathcal{X} ; \mathbb{Z} / \ell)
$$

is a quasi-isomorphism in the étale topology (where the left hand side means the associated complex of constant sheaves). Equivalently, for any $n \in \mathbb{N}$, the sheaf $H_{n}(\mathcal{X} ; \mathbb{Z} / \ell)$ is constant in the étale topology.

Finally the natural ring homomorphism (see below for the precise definition):

$$
H_{e t}^{*}(\mathcal{X} ; \mathbb{Z} / \ell) \rightarrow H^{*}(\mathcal{X}(k) ; \mathbb{Z} / \ell)
$$

is an isomorphism.

Proof. The first statement follows from the definition of the rigidity property at $\ell$, Lemma 2.11 above and Lemma 2.15 below, just by using the property of Definition 2.9 for henselization of closed points of smooth $k$-schemes.

The last assertion follows from this and the fact that if we let $\mathbb{Z} / \ell_{e t}(k)$ denote the abelian category of sheaves of $\mathbb{Z} / \ell$-modules $[\mathbf{2 3}]$, and $D\left(\mathbb{Z} / \ell_{e t}(k)\right)$ its derived category, then for any space $\mathcal{X}$, by the very definition of étale cohomology, one has a canonical isomorphism

$$
\operatorname{Hom}_{D\left(\mathbb{Z} / \ell_{e t}(k)\right)}\left(C_{*}(\mathcal{X} ; \mathbb{Z} / \ell)_{e t} ; \mathbb{Z} / \ell[*]\right) \cong H_{e t}^{*}(\mathcal{X} ; \mathbb{Z} / \ell)
$$

where $C_{*}(\mathcal{X} ; \mathbb{Z} / \ell)_{e t}$ is the simplicial mod $\ell$ chain complex of $\mathcal{X}$ in the étale topology, in other words the sheafification of $C_{*}(\mathcal{X} ; \mathbb{Z} / \ell)$ in the étale topology. The canonical (iso)morphism mentioned in the statement of the Theorem is thus the composition:

$$
\begin{aligned}
H_{e t}^{*}(\mathcal{X} ; \mathbb{Z} / \ell) & =\operatorname{Hom}_{D\left(\mathbb{Z} / \ell_{e t}(k)\right)}\left(C_{*}(\mathcal{X} ; \mathbb{Z} / \ell)_{e t} ; \mathbb{Z} / \ell[*]\right) \\
& \cong H_{D}\left(\mathbb{Z} / \ell_{e t}(k)\right) \\
& =H_{* t}^{*}(\mathcal{X}(k) ; \mathbb{Z} / \ell)=H^{*}(\mathcal{X}(k) ; \mathbb{Z} / \ell)
\end{aligned}
$$

induced by the (étale) quasi-isomorphism (2.1). The last identification $H_{e t}^{*}(\mathcal{X}(k) ; \mathbb{Z} / \ell)=H^{*}(\mathcal{X}(k) ; \mathbb{Z} / \ell)$ follows from the fact that any constant sheaf of $\mathbb{Z} / \ell$-modules in the étale topology is projective (as $k$ is algebraically closed).

REMARK 2.14. There is no obvious sheaf theoretic reformulation of the weak or strong rigidity property. For instance observe that the sheaf $\underline{\mathbf{K}}_{n}^{M}$, $n \geq 1$ say, has the strong rigidity property. In particular the associated sheaf to $\underline{\mathbf{K}}_{n}^{M} / \ell$ in the étale topology is indeed trivial (easy to check, any unit is an $\ell$-power locally in the étale toplogy). However the strong rigidity 
property tells us much more: for any henselization $\mathcal{O}_{X, x}^{h}$ of a point $x$ in a smooth $k$-scheme $X$ the morphism

$$
\underline{\mathbf{K}}_{n}^{M} / \ell\left(\mathcal{O}_{X, x}^{h}\right) \rightarrow \underline{\mathbf{K}}_{n}^{M} / \ell(\kappa(x))
$$

is an isomorphism.

We will only care on the rigidity property in this work as it is sufficient to prove Theorem 3 .

LEMma 2.15. Let $M$ be a sheaf of abelian group in the étale topology on $S m_{k}, k$ algebraically closed. Assume that for each henselization $\mathcal{O}_{X, x}^{h}$ of the local ring of a closed point $x \in X \in S m_{k}$ (with residue field automatically $k$ ), the morphism $M\left(\mathcal{O}_{X, x}^{h}\right) \rightarrow M(k)$ is an isomorphism. Then $M$ is a constant sheaf in the étale topology, that is to say the morphism $M(k)_{e t} \rightarrow M$ is an isomorphism.

Proof. The morphism $M(k)_{e t} \rightarrow M$ is an injective morphism because for $X$ irreducible smooth over $k$, the morphism $M(k)_{e t} \rightarrow M(X)$ can be identified with the morphism $M(k) \rightarrow M(X)$ induced by the structural morphism $X \rightarrow \operatorname{Spec}(k)$. Thus we may replace $M$ by the cokernel of this morphism and assume that $M(k)=0$. We thus have to prove that $M=0$. Take $\alpha \in M(X)$. For each closed point $x \in X(k), \alpha_{x}=0 \in M\left(\mathcal{O}_{X, x}^{h}\right)$ (because $M(k)=0$ ). This means that there is an étale morphism $V_{x} \rightarrow X$ whose image contains $x$ such that $\alpha \mid V_{x}=0$. But the family $\left\{V_{x} \rightarrow X\right\}_{x}$ is a covering family of $X$ in the étale topology, so that $\alpha=0$.

REMARK 2.16. 1) Let $\mathcal{X}$ be a pointed simply connected space. Let $\mathcal{X} \rightarrow$ $\mathcal{X}\left[\frac{1}{\ell}\right]$ be the universal morphism obtained by inverting $\ell$ in the homotopy sheaves of $\mathcal{X}$ (this is constructed as in [58]). The homotopy fiber $\mathcal{X}_{\ell-\text { tor }}$ of the morphism $\mathcal{X} \rightarrow \mathcal{X}\left[\frac{1}{\ell}\right]$ is a space whose homotopy sheaves are $\ell$-torsion. If $\mathcal{X}$ satisfies has the rigidity property at $\ell$, it is easy to check that $\mathcal{X}_{\ell-\text { tor }}$ also has the rigidity property at $\ell$. Moreover if $k$ is algebraically closed, then $\mathcal{X}_{\ell-\text { tor }}(k) \rightarrow \mathcal{X}$ is a simplicial weak equivalence in the étale topology. In other words, $\mathcal{X}_{\ell-\text { tor }}$ becomes constant in the étale topology.

2) One may deduce from the Theorem that if a space $\mathcal{X}$ has the rigidity property at $\ell$, the obvious morphism $\mathcal{X}(k) \rightarrow \mathcal{X}$ induces an equivalence of pro- $\ell$ spaces $\mathcal{X}(k)_{\ell} \rightarrow \hat{\mathcal{X}}_{\ell, e t}$ after taking the étale homotopy type functor $[\mathbf{3}, \mathbf{1 3}]$, where the left hand side means the pro- $\ell$-completion of the simplicial set $\mathcal{X}(k)$.

Taking Theorem 2.13 into account, we have reduced the proof of Theorem 1.2 and thus of Theorem 3 to the following:

THEOREM 2.17. For any perfect field $k$ and any split semi-simple $k$-group $G$, the space

$$
\operatorname{BSing}_{\bullet}^{\mathbb{A}^{1}}(G)
$$

has the rigidity property. 
REMARK 2.18. As we already mentioned, in fact, we may prove the strong rigidity property. See $[\mathbf{4 2}][\mathbf{4 3}]$.

Now to start proving Theorem 2.17, we need at last to let the $\mathbb{A}^{1}$-chain complex come in the game.

\section{The rigidity property and the $\mathbb{A}^{1}$-chain complex}

3.1. $\mathbb{A}^{1}$-chain complexes and the $\mathbb{A}^{1}$-lower central series. We want now to connect the rigidity property of a space $\mathcal{X}$, or equivalently (see the previous section) of its simplicial chain complex $C_{*}(\mathcal{X})$, to the rigidity property of its $\mathbb{A}^{1}$-chain complex $C_{*}^{\mathbb{A}^{1}}(\mathcal{X})$. The latter is still a mysterious object, obtained from $C_{*}(\mathcal{X})$ by applying a rather abstract functor $L_{\mathbb{A}^{1}}^{a b}$ of $\mathbb{A}^{1}$-localization of chain complexes. But on the other hand it appears also to be more flexible. First it is clear that the morphism

$$
C_{*}^{\mathbb{A}^{1}}(B G) \rightarrow C_{*}^{\mathbb{A}^{1}}\left(B \operatorname{Sing} \mathbb{A}^{1}(G)\right)
$$

is a quasi-isomorphism. At this point there is no difference between the two spaces involved. One of the crucial steps in our approach is to be able to observe that $C_{*}^{\mathbb{A}^{1}}(G)$, and then $C_{*}^{\mathbb{A}^{1}}\left(B \operatorname{Sing} g_{\bullet}^{\mathbb{A}^{1}}(G)\right)$, lies in the thick subcategory of chain complexes generated by the $C_{*}^{\mathbb{A}^{1}}\left(\left(\mathbb{G}_{m}\right)^{\wedge n}\right)$ 's, as it follows from the Bruhat decomposition. This wouldn't be possible if we would stay at the level of simplicial chain complexes.

We start by giving some recollections on $\mathbb{A}^{1}$-chain complexes from $[\mathbf{3 8}$, 41]. We let $D(\mathcal{A} b(k))$ be the derived category of $\mathcal{A} b(k)$, that is to say the category obtained from $\mathcal{C}_{*}(\mathcal{A} b(k))$ by inverting the quasi-isomorphisms. This category is the homotopy category of a model category structure [49] on $\mathcal{C}_{*}(\mathcal{A} b(k))$ with weak equivalences the quasi-isomorphisms and cofibrations the monomorphisms ${ }^{4}$. In the case of the category of abelian sheaves in the Nisnevich topology, which has a set of generators of finite cohomological dimension, this is well known, see [41, Section 3.2].

We let $D^{\mathbb{A}^{1}-l o c}(\mathcal{A} b(k)) \subset D(\mathcal{A} b(k))$ the full subcategory consisting of $\mathbb{A}^{1}$-local chain complexes as defined in [41, Section 3.2 p. 94]. From loc. cit. this inclusion admits a left adjoint called the $\mathbb{A}^{1}$-localization functor

$$
L_{\mathbb{A}^{1}}^{a b}: D(\mathcal{A} b(k)) \subset D_{\mathbb{A}^{1}}(\mathcal{A} b(k))
$$

which induces an equivalence of categories $D_{\mathbb{A}^{1}}(\mathcal{A} b(k)) \cong D^{\mathbb{A}^{1}-l o c}(\mathcal{A} b(k))$, where $D_{\mathbb{A}^{1}}(\mathcal{A} b(k))$ is the $\mathbb{A}^{1}$-derived category.

The main result of $[38]$ is that the functor $L_{\mathbb{A}^{1}}^{a b}$ preserves connectivity of chain complexes. As a consequence, we proved in loc. cit. that if $\mathcal{A} b_{\mathbb{A}^{1}}(k) \subset$ $\mathcal{A} b(k)$ denote the full subcategory of strictly $\mathbb{A}^{1}$-invariant sheaves, a chain

\footnotetext{
${ }^{4}$ The existence of this model category structure on the category of complexes in a Grothendieck abelian category is due independently to several authors, amongst them A. Joyal, and the author, but so far unpublished
} 
complex $C_{*}$ is $\mathbb{A}^{1}$-local if and only if its homology sheaves $H_{*}\left(C_{*}\right)$ are in $\mathcal{A} b_{\mathbb{A}^{1}}(k)$.

Remark 3.1. If $C_{*} \in \mathcal{C}_{*}(\mathcal{A} b(k))$ has trivial homology sheaves in degrees $\leq 0$, it is proven ${ }^{5}$ in $[\mathbf{4 1}$, Corollary 3.24 p. 100] that the morphism of spaces induced by the $\mathbb{A}^{1}$-localization functor $K C_{*} \rightarrow K L_{\mathbb{A}^{1}}^{a b} C_{*}$ is an $\mathbb{A}^{1}$ weak equivalence to an $\mathbb{A}^{1}$-local space. Thus in that case the canonical morphism

is a simplicial weak equivalence.

$$
L_{\mathbb{A}^{1}} K C_{*} \rightarrow K L_{\mathbb{A}^{1}}^{a b} C_{*}
$$

However in general (if $H_{0} C_{*} \neq 0$ ) this is not true, and for this reason we used different notations for the $\mathbb{A}^{1}$-localization functor for spaces and for complexes. However in each of the situations we will meet the previous result will imply that we may identify those using the previous simplicial weak-equivalence. We will use frequently this identification.

Let $\mathcal{X}$ be a space and let $C_{*}(\mathcal{X} ; \mathbb{Z})$ be its normalized chain complex, see Section 2.2. The $\mathbb{A}^{1}$-localization $L_{\mathbb{A}^{1}}^{a b}\left(C_{*}(\mathcal{X} ; \mathbb{Z})\right)$ is denoted by $C_{*}^{\mathbb{A}^{1}}(\mathcal{X} ; \mathbb{Z})$, or sometimes simply $C_{*}^{\mathbb{A}^{1}}(\mathcal{X})$, and is called the $\mathbb{A}^{1}$-chain complex of $\mathcal{X}$.

From the previous results, it follows that the homology sheaves $H_{i}^{\mathbb{A}^{1}}$ $(\mathcal{X} ; \mathbb{Z})$ of $C_{*}^{\mathbb{A}^{1}}(\mathcal{X} ; \mathbb{Z})$ are strictly $\mathbb{A}^{1}$-invariant sheaves and vanish for $i<0$. This are called the $\mathbb{A}^{1}$-homology sheaves of the space $\mathcal{X}$.

Remark 3.2. Even if $\mathcal{X}$ is an $\mathbb{A}^{1}$-local space, the chain complex $C_{*}(\mathcal{X} ; \mathbb{Z})$ has no reason to be $\mathbb{A}^{1}$-local! See Remark 3.11 below.

When $\mathcal{X}$ is pointed, one may consider the reduced $\mathbb{A}^{1}$-chain complex $\tilde{C}_{*}^{\mathbb{A}^{1}}(\mathcal{X} ; \mathbb{Z})$, as well the reduced $\mathbb{A}^{1}$-homology sheaves $\tilde{H}_{*}^{\mathbb{A}^{1}}(\mathcal{X} ; \mathbb{Z})$, by removing the factor $\mathbb{Z}$ in $C_{*}^{\mathbb{A}^{1}}(\mathcal{X} ; \mathbb{Z})$ corresponding to the base point. We may also consider as in the simplicial case the $\mathbb{A}^{1}$-chain complexes and homology sheaves with $\mathbb{Z} / \ell$-coefficients: $C_{*}^{\mathbb{A}^{1}}(\mathcal{X} ; \mathbb{Z} / \ell)$ and $H_{*}^{\mathbb{A}^{1}}(\mathcal{X} ; \mathbb{Z} / \ell)$.

Now we introduce one of the main new technical tool: the $\mathbb{A}^{1}$-lower central series. Let us start by recalling the classical one.

The lower central series for sheaves of groups. We assume the reader familiar with the usual lower central series for groups, which can be found for instance in $[\mathbf{9}]$. Let $G$ be a sheaf of groups $\left(\right.$ on $\left.\left(S m_{k}\right)_{N i s}\right)$. Its "naive" lower central series ... $\Gamma_{r} G \subset \Gamma_{r-1} G \subset \Gamma_{1} G=G$ is defined by sheafification of the usual one on $G$. It is the smallest descending filtration $\cdots \subset \Gamma_{r+1} \subset \Gamma_{r} \subset \cdots \subset G$ of $G$ by normal subgroups such that $\left[G, \Gamma_{r}\right] \subset$ $\Gamma_{r+1}$.

It is well known ( $c f$ loc. cit.) that there is a structure of graded Lie algebra on the associated graded (sheaf of) abelian group(s) $G r_{*}(G)=$ $\oplus \Gamma_{r}(G) / \Gamma_{r+1}(G)$, that $G r_{1}(G)=G_{a b}$ the abelianization of $G$, and that the induced morphism

$$
L_{*}\left(G_{a b}\right) \rightarrow G r_{*}(G)
$$

\footnotetext{
${ }^{5}$ This is at this place that we need for the moment that $\operatorname{char}(k) \neq 2$.
} 
from the free graded Lie algebra $L_{*}\left(G_{a b}\right)$ is an epimorphism, and an isomorphism for $G$ stalkwise free, see Theorem 7.5 of $[\mathbf{9}]$.

The Kan construction $G(\mathcal{X})$. Let $\mathcal{X}$ be a reduced pointed space, meaning that $\mathcal{X}_{0}$ is equal to the point. One denotes by $G(\mathcal{X})$ the sheafification of the Kan $G$ construction [9, Definition 3.15 p. 133] on the presheaf $U \mapsto G(\mathcal{X}(U))$. It is a simplicial sheaf of groups, stalkwise free.

Standard homotopical algebra provides a canonical isomorphism in the pointed simplicial homotopy category of spaces between $R \Omega(\mathcal{X})$ and $G(\mathcal{X})$.

Using the main results of $[\mathbf{8}]$ we get:

THEOREM 3.3. Let $\mathcal{X}$ be a simplicially 1 -connected reduced pointed space. Then for each $r \geq 1$ the morphism

$$
G(\mathcal{X}) \rightarrow G(\mathcal{X}) / \Gamma_{r}(G(\mathcal{X}))
$$

is at least $\left\{1+\log _{2}(r)\right\}$ simplicially connected.

The fiber of the morphism $G(\mathcal{X}) / \Gamma_{r}(G(\mathcal{X})) \rightarrow G(\mathcal{X}) / \Gamma_{r-1}(G(\mathcal{X}))$ is of course isomorphism as a simplicial sheaf of groups to $\Gamma_{r-1}(G(\mathcal{X})) / \Gamma_{r}(G(\mathcal{X}))$. From what we recall above, $G(\mathcal{X})$ being stalkwise free, this simplicial abelian group is isomorphic to the simplicial sheaf of abelian groups

$$
F_{r}:=L_{r-1}\left(G(\mathcal{X})_{a b}\right)
$$

obtained by degreewise applying the free Lie algebra functor in degree $r-1$ to the simplicial abelian group $G(\mathcal{X})_{a b}$. This simplicial sheaf of abelian groups $F_{r}$ is also (approximatively) $\left\{1+\log _{2}(r)\right\}$ simplicially connected.

The $\mathbb{A}^{1}$-lower central series. We may now deduce from the unstable $\mathbb{A}^{1}$-connectivity result of $[\mathbf{4 1}]$ the following:

TheOREm 3.4. Let $\mathcal{X}$ be a 1-connected $\mathbb{A}^{1}$-local reduced pointed space. Then for each $r \geq 1$ the morphism

$$
G(\mathcal{X}) \cong L_{\mathbb{A}^{1}}(G(\mathcal{X})) \rightarrow L_{\mathbb{A}^{1}}\left(G(\mathcal{X}) / \Gamma_{r}(G(\mathcal{X}))\right)
$$

is $\left\{1+\log _{2}(r)\right\}$-connected.

Proof. From [41] the exact sequence of simplicial sheaf of groups $\Gamma_{r}(G(\mathcal{X})) \rightarrow G(\mathcal{X}) \rightarrow G(\mathcal{X}) / \Gamma_{r}(G(\mathcal{X}))$ is automatically an $\mathbb{A}^{1}$-fibration sequence. Now from loc. cit. the space $\Gamma_{r}(G(\mathcal{X}))$ being simplicially $\{1+$ $\left.\log _{2}(r)\right\}$ connected, is $\left\{1+\log _{2}(r)\right\} \mathbb{A}^{1}$-connected.

For a reduced Kan simplicial set $K$ recall $[9$, p. 125] that its "path complex" $\mathcal{P} K$ is the simplicial set with $\mathcal{P} K_{n}:=K_{n+1}$ and $d_{i}:=d_{i}+1$, $s_{i}:=s_{i+1}$. The morphism $d_{0}$ induces a Kan fibration $\mathcal{P} K \rightarrow K$ with fiber $\Omega K$ the loop space of $K$. This construction generalizes to reduced simplicial sheaf of sets.

If $\mathcal{X}$ is a pointed space, we let $\tilde{\mathbb{Z}}(\mathcal{X})$ be the free sheaf of abelian groups generated by $\mathcal{X}$ modulo the relation $*=0$ (base point equals 0 ). In other 
words we have a canonical direct sum decomposition in the category of simplicial sheaves of abelian groups of the form:

$$
\mathbb{Z}(\mathcal{X})=\mathbb{Z} \oplus \tilde{\mathbb{Z}}(\mathcal{X})
$$

The associated normalized chain complex to the simplicial sheaf of abelian groups $\mathbb{Z}(\mathcal{X})$ is called the reduced chain complex of $\mathcal{X}$ and is denoted by $\tilde{C}_{*}(\mathcal{X})$ of $\mathcal{X}$. The Dold-Kan correspondance gives an isomorphism of simplicial sheaves of abelian groups (see [9])

$$
\tilde{\mathbb{Z}}(\mathcal{X}) \cong K\left(\tilde{C}_{*}(\mathcal{X})\right)
$$

An easy inspection in the previous definitions gives the following standard fact:

LEMMA 3.5. There exists canonical isomorphisms of simplicial sheaves of abelian groups

$$
G(\mathcal{X})_{a b} \cong \Omega(\tilde{\mathbb{Z}}(\mathcal{X})) \cong \Omega K\left(\tilde{C}_{*}(\mathcal{X})\right) \cong K\left(\tilde{C}_{*}(\mathcal{X})[-1]\right)
$$

It follows that the space $F_{s}$ considered above is in fact (up to canonical isomorphism) $F_{s}=L_{s-1}\left(K\left(\tilde{C}_{*}(\mathcal{X})[-1]\right)\right)$, a functor of the reduced simplicial chain complex $\tilde{C}_{*}(\mathcal{X})$. Let us denote by $F_{s}^{\mathbb{A}^{1}}$ the $\mathbb{A}^{1}$-localization of $F_{s}$. We will use the simplicial fibration sequences

$$
F_{s}^{\mathbb{A}^{1}} \rightarrow L_{\mathbb{A}^{1}}\left(G(\mathcal{X}) / \Gamma_{s}(G(\mathcal{X}))\right) \rightarrow L_{\mathbb{A}^{1}}\left(G(\mathcal{X}) / \Gamma_{s-1}(G(\mathcal{X}))\right)
$$

It is (up to simplicial weak equivalence) a principal fibration sequence with connected structural group

$$
F_{s}^{\mathbb{A}^{1}} \cong L_{\mathbb{A}^{1}}\left(L_{s-1}\left(K\left(\tilde{C}_{*}(\mathcal{X})[-1]\right)\right)\right)
$$

An $\mathbb{A}^{1}$-quasi-isomorphism $C_{*} \rightarrow D_{*}$ is a morphism of chain complexes which induces an isomorphism in the $\mathbb{A}^{1}$-derived category $D_{\mathbb{A}^{1}}(\mathcal{A} b(k))$ or, equivalently, which induces a quasi-isomorphism after applying the $\mathbb{A}^{1}$ localization functor $L_{\mathbb{A}^{1}}^{a b}$. For instance $C_{*} \rightarrow L_{\mathbb{A}^{1}}^{a b}\left(C_{*}\right)$ is an $\mathbb{A}^{1}$-quasi isomorphism. The following result may be proven along the "classical" lines (see [11]) for instance.

Theorem 3.6. Let $s \geq 1$ be an integer and let $\phi: C_{*} \rightarrow D_{*}$ be a morphism of chain complexes both concentrated in positive degrees (i.e. with $H_{i}\left(C_{*}\right)=0$ for $i \leq 0$. If the morphism $\phi$ is an $\mathbb{A}^{1}$-quasi-isomorphism, that is to say, then for any $s \geq 1$ the morphism of spaces

$$
L_{s}(\phi): L_{s}\left(K C_{*}\right) \rightarrow L_{s}\left(K D_{*}\right)
$$

is an $\mathbb{A}^{1}$-equivalence (of spaces).

For this reason the chain complexes (or spaces) of the form $L_{\mathbb{A}^{1}}$ $\left(L_{s}\left(K C_{*}\right)\right)$ are called the total derived functors of the (non-additive) functor $L_{s}$. 
Thus it follows that the above space $F_{s}^{\mathbb{A}^{1}} \cong L_{\mathbb{A}^{1}}\left(L_{s-1}\left(K\left(\tilde{C}_{*}(\mathcal{X})[-1]\right)\right)\right)$ is simplicially weakly equivalent to

$$
F_{s}^{\mathbb{A}^{1}} \cong L_{\mathbb{A}^{1}}\left(L_{s-1}\left(K\left(\tilde{C}_{*}^{\mathbb{A}^{1}}(\mathcal{X})[-1]\right)\right)\right)
$$

The right hand side is called the total $\mathbb{A}^{1}$-derived functor of $L_{s-1}$ on $\tilde{C}_{*}^{\mathbb{A}^{1}}$ $(\mathcal{X})[-1])$, see below for some properties of these.

Together with what we have done before, we may summarize the previous results as follows. Let $\mathcal{X}$ be a 1 -connected $\mathbb{A}^{1}$-local reduced pointed space. For each $s \geq 1$ set

$$
W_{s}(\mathcal{X}):=B\left(L_{\mathbb{A}^{1}}\left(G(\mathcal{X}) / \Gamma_{s}(G(\mathcal{X}))\right)\right) \cong L_{\mathbb{A}^{1}}\left(B\left(G(\mathcal{X}) / \Gamma_{s}(G(\mathcal{X}))\right)\right)
$$

and $\left.\gamma_{s}(\mathcal{X}):=L_{\mathbb{A}^{1}} B \Gamma_{s}(G(\mathcal{X}))\right)$ the homotopy fiber of the canonical morphism $\mathcal{X} \rightarrow W_{s}(\mathcal{X})$ (in the simplicial homotopy category).

TheOREm 3.7. Let $\mathcal{X}$ be a 1-connected $\mathbb{A}^{1}$-local reduced pointed space. Then for any $s \geq 1$ the $\mathbb{A}^{1}$-local space $\gamma_{s}(\mathcal{X})$ is $\left\{1+\log _{2}(s)\right\}$-connected and in particular the morphism

$$
\mathcal{X} \rightarrow W_{s}(\mathcal{X})
$$

is $\left\{1+\log _{2}(s)\right\}-\mathbb{A}^{1}$-connected.

Moreover the morphism $W_{s} \rightarrow W_{s-1}$ a principal fibration with group

$$
B F_{s}^{\mathbb{A}^{1}} \cong B L_{\mathbb{A}^{1}}\left(L_{s-1}\left(K\left(\tilde{C}_{*}(\mathcal{X})[-1]\right)\right)\right) \cong B L_{\mathbb{A}^{1}}\left(L_{s-1}\left(K\left(\tilde{C}_{*}^{\mathbb{A}^{1}}(\mathcal{X})[-1]\right)\right)\right)
$$

Clearly it follows:

Corollary 3.8. Let $\mathcal{X}$ be a 1-connected $\mathbb{A}^{1}$-local pointed space. If for each $s \geq 1$ the space $B L_{\mathbb{A}^{1}}\left(L_{s-1}\left(K\left(\tilde{C}_{*}^{\mathbb{A}^{1}}(\mathcal{X})[-1]\right)\right)\right)$ has the rigidity property, then $\mathcal{X}$ has the rigidity property.

The rigidity property of the space $B L_{\mathbb{A}^{1}}\left(L_{s-1}\left(K\left(\tilde{C}_{*}^{\mathbb{A}^{1}}(\mathcal{X})[-1]\right)\right)\right)$ is a property concerning only the chain complex $\tilde{C}_{*}^{\mathbb{A}^{1}}(\mathcal{X})$. We thus have reduced the rigidity property for such 1 -connected $\mathbb{A}^{1}$-local pointed spaces $\mathcal{X}$ to a property involving only the reduced $\mathbb{A}^{1}$-chain complex $\tilde{C}_{*}^{\mathbb{A}^{1}}(\mathcal{X})$. In the next section we make this observation quite precise.

\section{2. $\mathbb{A}^{1}$-homological characterization of the rigidity property.}

$\mathbb{A}^{1}$-Derived functor of the free Lie algebra functor. Our aim here is to prove the following Theorem, mentioned has Theorem 10 in the introduction:

TheOREM 3.9. Let $\mathcal{X}$ be a pointed simply-connected $\mathbb{A}^{1}$-local space over the base field $k$, $\ell$ be a prime different from char $(k)$. If the $\mathbb{A}^{1}$-chain complexes $C_{*}^{\mathbb{A}^{1}}\left(\mathcal{X}^{n} ; \mathbb{Z} / \ell\right), n \geq 1$, have the rigidity property at $\ell$, then the space $\mathcal{X}$ satisfies the rigidity property at $\ell$.

To explain the fineness of the previous Theorem 3.9 let us make some observations. 
REMARK 3.10. We already observed that there exists an $\mathbb{A}^{1}$-localization functor $L_{\mathbb{A}^{1}}$ on spaces which commutes to finite products, thus takes simplicial sheaves of $\mathbb{Z} / \ell$-modules to simplicial sheaves of $\mathbb{Z} / \ell$-modules. This follows from [44] Theorem 1.66 p. 69 and Lemma 3.20 p. 93.

Given a chain complex of sheaves of abelian groups $C_{*}$ concentrated in non negative degrees, the associated morphism of normalized chain complexes $C_{*} \rightarrow\left(L_{\mathbb{A}^{1}}\left(K C_{*}\right)\right)_{*}^{N}$ is an $\mathbb{A}^{1}$-quasi-isomorphism, but in general $\left(L_{\mathbb{A}^{1}}\left(K C_{*}\right)\right)_{*}^{N}$ is not an $\mathbb{A}^{1}$-local complex in the sense of $[\mathbf{4 1}]$.

It is a non-trivial result $\left[\mathbf{4 1}\right.$, Corollary 3.24] that for a complex $C_{*} \in$ $\mathcal{C}_{*}^{\geq 0}(k)$ which is 0 -connected, the morphism

$$
K C_{*} \rightarrow K\left(L_{\mathbb{A}^{1}}^{a b} C_{*}\right)
$$

is an $\mathbb{A}^{1}$-equivalence of spaces, which implies that the chain $\left(L_{\mathbb{A}^{1}}\left(K C_{*}\right)\right)_{*}^{N}$ is in that case canonically isomorphic to $L_{\mathbb{A}^{1}}^{a b}\left(C_{*}\right)$ in $D(\mathcal{A} b(k))$. Quickly said, we have a simplicial weak-equivalence

$$
L_{\mathbb{A}^{1}}\left(K C_{*}\right) \cong K\left(L_{\mathbb{A}^{1}}^{a b} C_{*}\right)
$$

This is false in general if $C_{*}$ is not assumed to be 0-connected. For instance take $C_{*}=\mathbb{Z}\left(\mathbb{G}_{m}\right)$. Then $\mathbb{Z}\left(\mathbb{G}_{m}\right)=K\left(\mathbb{Z}\left(\mathbb{G}_{m}\right)\right) \rightarrow K\left(L_{\mathbb{A}^{1}}^{a b} \mathbb{Z}\left(\mathbb{G}_{m}\right)\right)$ is not an $\mathbb{A}^{1}$-equivalence. This follows from the computations of loc. cit. and the fact that the sheaf (and thus the space) $\mathbb{Z}\left(\mathbb{G}_{m}\right)$ is $\mathbb{A}^{1}$-invariant and thus $\mathbb{A}^{1}$-local. If the previous morphism were an $\mathbb{A}^{1}$-equivalence it would thus be a simplicial weak-equivalence (between two $\mathbb{A}^{1}$-local spaces) but from [41, Theorem 2.37], the sheaf $\pi_{0}^{\mathbb{A}^{1}}$ of the space $K\left(L_{\mathbb{1}^{1}}^{a b} \mathbb{Z}\left(\mathbb{G}_{m}\right)\right)$ is the sheaf $\underline{\mathbf{K}}_{1}^{M W}$ and the induced morphism in degree $0, \mathbb{Z}\left(\mathbb{G}_{m}\right) \rightarrow \underline{\mathbf{K}}_{1}^{M W}$ is not an isomorphism.

REMARK 3.11. Let $\mathcal{X}$ be a space. The canonical morphism of chain complexes

$$
C_{*}(\mathcal{X}) \rightarrow C_{*}^{\mathbb{A}^{1}}(\mathcal{X})
$$

from the simplicial chain complex to the $\mathbb{A}^{1}$-local chain complex of $\mathcal{X}$ is not in general a quasi-isomorphism. Of course by the very definition it is an $\mathbb{A}^{1}$ quasi isomorphism, as $C_{*}^{\mathbb{A}^{1}}(\mathcal{X})$ is the $\mathbb{A}^{1}$-localization of $C_{*}(\mathcal{X})$. But even if $\mathcal{X}$ is $\mathbb{A}^{1}$-local, $C_{*}(\mathcal{X}) \rightarrow C_{*}^{\mathbb{A}^{1}}(\mathcal{X})$ is also alsmost never a quasi-isomorphism! In other words in general, the homology sheaves of $C_{*}(\mathcal{X})$ will not be strictly $\mathbb{A}^{1}$-invariant even if $\mathcal{X}$ itself is $\mathbb{A}^{1}$-local.

For instance take $\mathcal{X}$ to be $B \mathbb{G}_{m}$, an $\mathbb{A}^{1}$-local space. Then $H_{1}\left(B \mathbb{G}_{m}\right)=$ $\mathbb{G}_{m}$ and the morphism $C_{*}\left(B \mathbb{G}_{m}\right) \rightarrow C_{*}^{\mathbb{A}^{1}}\left(B \mathbb{G}_{m}\right)$ is an isomorphism in homology up to degree 1 included. But $H_{2}\left(B \mathbb{G}_{m}\right)=\Lambda_{2}\left(\mathbb{G}_{m}\right)$ can be shown not to be a strictly $\mathbb{A}^{1}$-invariant sheaf; as a consequence, the previous morphism of chain complexes is not an isomorphism on $\mathrm{H}_{2}$.

One should also notice that a product of finitely many spaces whith the rigidity property has the rigidity property, and a (derived) tensor product of finitely many chain complexes with the rigidity property has the rigidity 
property, as it follows from the Künneth formula. However, a tensor product of finitely many $\mathbb{A}^{1}$-local chain complexes will not be in general $\mathbb{A}^{1}$-local.

We end up this remark by the following questions: let $\mathcal{X}$ be a space with the rigidity property is it true that its $\mathbb{A}^{1}$-localization $L_{\mathbb{A}^{1}}(\mathcal{X})$ also has the rigidity property ? Let $C_{*}$ be a chain complex with the rigidity property. Is it true that its $\mathbb{A}^{1}$-localization $L_{\mathbb{A}^{1}}^{a b}\left(C_{*}\right)$ will have the rigidity property?

Now we sketch the proof of Theorem 3.9. We first observe that to prove Theorem 3.9 it suffices to establish:

THEOREM 3.12. Let $C_{*}$ be a chain complex of $\mathbb{Z} / \ell$-modules such that $H_{i}\left(C_{*}\right)=0$ for $i \leq 0$ and such that for each integer $n \geq 1$ the $\mathbb{A}^{1}$-localization $L_{\mathbb{A}^{1}}^{a b}\left(\left(C_{*}\right)^{\otimes n}\right)$ has the rigidity property at $\ell$. Then for each $s \geq 1$ the space

$$
L_{\mathbb{A}^{1}}\left(L_{s}\left(K\left(C_{*}\right)\right)\right.
$$

has the rigidity property at $\ell$.

Proof of Theorem 3.9 using Theorem 3.12. By Corollary 3.8 it is sufficient to show that the spaces $B L_{\mathbb{A}^{1}}\left(L_{s}\left(K\left(\tilde{C}_{*}^{\mathbb{A}^{1}}(\mathcal{X})[-1]\right)\right)\right)$ have the rigidity property at $\ell$, or equivalently that the spaces $L_{\mathbb{A}^{1}}\left(L_{s}\left(K\left(\tilde{C}_{*}^{\mathbb{A}^{1}}\right.\right.\right.$ $(\mathcal{X})[-1]))$ ) have the rigidity property at $\ell$ by Remark 2.5 . By Theorem 3.6, we know that

$$
L_{\mathbb{A}^{1}}\left(L_{s}\left(K\left(\tilde{C}_{*}(\mathcal{X})[-1]\right)\right)\right) \rightarrow L_{\mathbb{A}^{1}}\left(L_{s}\left(K\left(\tilde{C}_{*}^{\mathbb{A}^{1}}(\mathcal{X})[-1]\right)\right)\right)
$$

is a simplicial weak equivalence, as well as

$$
L_{\mathbb{A}^{1}}\left(L_{s}\left(K\left(\tilde{C}_{*}(\mathcal{X} ; \mathbb{Z} / \ell)[-1]\right)\right)\right) \rightarrow L_{\mathbb{A}^{1}}\left(L_{s}\left(K\left(\tilde{C}_{*}^{\mathbb{A}^{1}}(\mathcal{X} ; \mathbb{Z} / \ell)[-1]\right)\right)\right)
$$

Now we see that it suffices to check that the spaces $L_{\mathbb{A}^{1}}\left(L_{s}\left(K\left(\tilde{C}_{*}^{\mathbb{A}^{1}}(\mathcal{X} ; \mathbb{Z} / \ell)\right.\right.\right.$ $[-1]))$ ) have the rigidity property at $\ell$. Now this follows from Theorem 3.12 applied to the chain complex $\left.C_{*}=\tilde{C}_{*}^{\mathbb{A}^{1}}(\mathcal{X} ; \mathbb{Z} / \ell)[-1]\right)$; observe that the $\mathbb{A}^{1}$-localization of the chain complexes $\left.\left(\tilde{C}_{*}^{\mathbb{A}^{1}}(\mathcal{X} ; \mathbb{Z} / \ell)[-1]\right)\right)^{\otimes n}$ is quasiisomorphic to the chain complex $\left.\tilde{C}_{*}^{\mathbb{A}^{1}}\left(\mathcal{X}^{\wedge n} ; \mathbb{Z} / \ell\right)[-n]\right)$, which, being a summand of $\left.C_{*}^{\mathbb{A}^{1}}\left(\mathcal{X}^{n} ; \mathbb{Z} / \ell\right)[-n]\right)$ has the rigidity property by assumptions, which shows that $C_{*}$ satisfies the assumption of Theorem 3.12.

Now to get Theorem 3.12 we use some standard technics of devissage together with the following remarks.

From $[\mathbf{9}, \mathbf{5 1}]$ the free Lie algebra of a direct sum $V_{1} \oplus V_{2}$ decomposes canonically as:

$$
\oplus_{I \in \mathcal{B}} L_{*}\left(V_{I}\right) \cong L_{*}\left(V_{1} \oplus V_{2}\right)
$$

where the index set $\mathcal{B}$ is the set of basic commutators $\mathcal{B}$ in two letters. This means in particular that the above isomorphism is an isomorphism of functors. This fact allows one for instance to control the statement of Theorem 3.12 if we have a cofibration sequence of chain complexes.

Let us recall some notations for functors $\mathcal{A} b \rightarrow \mathcal{A} b$. $T_{n}$ will denote the $n$-th tensor product functor $A \mapsto A^{\otimes n}, S_{n}$ will denote the $n$-th symmetric tensor product functor $A \mapsto A^{\otimes n} / \Sigma_{n}$. We will also have to use the $\ell$-cyclic 
product functor $A \mapsto C_{\ell}(A):=A^{\otimes \ell} / \mathbb{Z} / \ell$ and more generally for $H \subset S_{m}$ a subgroup, we denote by $S_{m}^{H}$ the functor $A \mapsto A^{\otimes n} / H$. Observe that if $\left[S_{m}: H\right]$ is prime to $\ell$, the epimorphism $S_{m}^{H} \rightarrow S_{m}$ admits a section when restricted (as functor) to the category of $\mathbb{Z} / \ell$-modules. We extend these notations in the obvious way to sheaves of abelian groups.

The remaining idea to prove the Theorem 3.12 is the following. Let $C_{*}$ be a chain complex of $\mathbb{Z} / \ell$-modules such that $H_{i}\left(C_{*}\right)=0$ for $i \leq 0$ and such that for each integer $n \geq 1$ the $\mathbb{A}^{1}$-localization $L_{\mathbb{A}^{1}}^{a b}\left(\left(C_{*}\right)^{\otimes n}\right)$ has the rigidity property. We deduce from this that the $\mathbb{A}^{1}$-localization $L_{\mathbb{A}^{1}}^{a b}\left(S_{n}\left(\left(C_{*}\right)\right)\right.$ have the rigidity property by some standard homological algebra step. One proceed by induction on $n$. Then one observe that if $\ell$ divides $n$ the functor $S_{n}$ can be expressed in term of functors $S_{n^{\prime}}$ with $n^{\prime}<n$ and the cyclic functor $C_{\ell}$. This one is understood in terms of the cohomology of $\mathbb{Z} / \ell$ with values in $T_{\ell}$.

Now we still have to deduce from these fact the rigidity property for the $L_{\mathbb{A}^{1}}^{a b}\left(L_{n}\left(C_{*}\right)\right)$ 's. The last idea is to use the Poincaré-Birkhoff-Witt Theorem. For $M$ a $\mathbb{Z} / \ell$-module, the isomorphism $L_{1}(M)=T_{1}(M)=M$ extends to a canonical morphism $L_{*}(M) \rightarrow T_{*}(M)$, obtained by considering the tensor algebra as a Lie algebra. This morphism is injective and more precisely, let $U_{i}(M)_{*} \subset T_{*}(M)$ be the sub-ZZ/ $/$-module generated by the products of $j$-elements of $L_{*}(M)$ for each $j \leq i$. One thus gets an increasing filtration of $\mathbb{N}$-graded $\mathbb{Z} / \ell$-modules

$$
L_{*}(M)=U_{1}(M)_{*} \subset U_{2}(M)_{*} \subset \cdots \subset U_{i}(M)_{*} \subset \cdots \subset T_{*}(M)
$$

The Poincaré-Birkhoff-Witt Theorem [4] asserts that the canonical morphism of commutative graded (by $i$ ) algebras $\oplus_{i} S_{i}\left(L_{*}(M)\right) \rightarrow \oplus_{i} U_{i}(M)_{*} /$ $U_{i-1}(M)_{*}$ is an isomorphism.

In a given degree $s$ this gives an increasing filtration

$$
L_{s}(M)=U_{1}(M)_{s} \subset U_{2}(M)_{s} \subset \cdots \subset U_{s-1}(M)_{s} \subset U_{s}(M)=T_{s}(M)
$$

such that the quotient $U_{i}(M)_{s} / U_{i-1}(M)$ is canonically isomorphic to the degree $i$ part of the symmetric algebra on the free Lie algebra over $M$. Using the usual formula $S_{*}(M \oplus N)=S_{*}(M) \otimes S_{*}(N)$, we see that for $i \geq 2, U_{i}(M)_{s} / U_{i-1}(M)_{s}$ is as functor equal to the finite direct sum of the functors of the form $M \mapsto S_{j_{1}}\left(L_{t_{1}}(M)\right) \otimes \cdots \otimes S_{j_{a}}\left(L_{t_{a}}(M)\right)$ with $\Sigma j_{b} t_{b}=s$ and $\Sigma j_{b}=i$.

This permits to finish the proof of Theorem 3.12 by induction on $s$. More details may be found in $[\mathbf{4 2}]$.

4. Reduction to the rigidity property for the $C_{*}^{\mathbb{A}^{1}}\left(\left(\mathbb{G}_{m}\right)^{\wedge n}\right)$ 's

\section{1. $\mathbb{A}^{1}$-chain complex of mixed Tate type.}

Definition 4.1. A full subcategory $\mathcal{C} \subset D_{\mathbb{A}^{1}}^{\geq 0}(k)$ is called a Class of complexes if the following properties hold:

(1) $\mathcal{C}$ is stable under arbitrary direct sums (and thus contains the 0 complex); 
(2) For any exact triangle $A \rightarrow B \rightarrow C \rightarrow A[1]$ in $D_{\mathbb{A}^{1}}^{\geq 0}(k)$, if $A \in \mathcal{C}$ and $(B \in \mathcal{C})$ then $(C \in \mathcal{C})$.

The class $\mathcal{C}$ is said to be multiplicative if moreover:

(3) For any $A \in \mathcal{C}$ and $(B \in \mathcal{C})$ then $A \otimes B \in \mathcal{C}$.

We will say that a full subcategory $\mathcal{C} \subset D_{\mathbb{A}^{1}}^{\geq 0}(k)$ is a strict Class of complexes if the following axiom holds in place of $(1)$ :

$\left(1^{\prime}\right) \mathcal{C}$ is stable under finite direct sums (and thus contains the 0 complex) and by direct summand.

Observe that a class is stable by telescopes and in particular by direct summand. Also classes and strict classes are stable by suspension.

EXAMPLE 4.2. If $\mathcal{C} \subset \mathcal{A} b_{\mathbb{A}^{1}}(k)$ is a Serre class in the abelian category $\mathcal{A} b_{\mathbb{A}^{1}}(k)$ in the usual sense then the full subcategory $D^{\mathcal{C}} \subset D_{\mathbb{A}^{1}}^{\geq 0}(k)$ consisting of chain complex with homology objects in $\mathcal{C}$ is a class of complexes in the previous sense.

EXAMPLE 4.3. The full subcategory $D_{\mathbb{A}^{1}}(\mathcal{A} b(k)) \subset D(\mathcal{A} b(k))$ consisting of $\mathbb{A}^{1}$-local chain complexes is a class of complex. Observe however it is not multiplicative.

EXAMPLE 4.4. An intersection of a family of classes is also a class of complexes.

Let $\left(C_{*}(i)\right)_{i}$ be a family of chain complexes in $D_{\mathbb{A}^{1}}^{\geq 0}(k)$. We let $\mathcal{C}\left(\left\{C_{*}(i)\right\}_{i}\right)$ $\subset D_{\mathbb{A}^{1}}^{\geq 0}(k)$ be the class generated by the $C_{*}(i)$, i.e. the intersection of all classes of complexes which contains this family. We may also introduce the strict class of complexes generated by a family of complexes.

Define by induction on $n$ an increasing family of full subcategories $\mathcal{C}^{(0)} \subset$ $\mathcal{C}^{(1)} \subset \ldots \mathcal{C}^{(n)} \cdots \subset D_{\mathbb{A}^{1}}^{\geq 0}(k)$ as follows: $\mathcal{C}^{(0)}$ is the full subcategory with objects the elements of the family $\left(C_{*}(i)\right)_{i}$. The category $\mathcal{C}^{(n)}$ has for objects the factors of the objects of $\mathcal{C}^{(n-1)}$ and the cones of morphisms in $\mathcal{C}^{(n-1)}$. Clearly

$$
\bigcup_{n} \mathcal{C}^{(n)}=\mathcal{C}\left(\left\{C_{*}(i)\right\}_{i}\right) \subset D_{\mathbb{A}^{1}}^{\geq 0}(k)
$$

ExAmple 4.5. If $\mathcal{C} \subset D^{\geq 0}(\mathcal{A} b(k))$ is a class of complexes, the full subcategory $\mathcal{C}_{\mathbb{A}^{1}} \subset D^{\geq 0}(\mathcal{A} b(k))$ consisting of complexes $C_{*}$ whose $\mathbb{A}^{1}$-localization $L_{\mathbb{A}^{1}}^{a b}\left(C_{*}\right)$ is in $\mathcal{C}$ is a class of complexes: this follows easily from the fact that $L_{\mathbb{A}^{1}}^{a b}(-)$ is exact (and preserves connectivity).

ExAMPLE 4.6. The full subcategory $\mathcal{C}^{\text {rig, } \ell, \geq 0} \subset D^{\geq 0}(\mathcal{A} b(k))$ consisting of $\mathbb{A}^{1}$-local chain complexes which have the rigidity property at $\ell$ is a class of complexes. This follows from Lemma 2.4.

It follows from Example 4.5 that the full subcategory $\mathcal{C}_{\mathbb{A}^{1}}^{\text {rig, } \ell, \geq 0} \subset D^{\geq 0}$ $(\mathcal{A} b(k))$ consisting of chain complexes whose $\mathbb{A}^{1}$-localization has the rigidity property at $\ell$ is a class of complexes. 
The usual splitting of the sheaf $\mathbb{Z}\left(\left(\mathbb{G}_{m}\right)^{n}\right)$ as

$$
\mathbb{Z}\left(\left(\mathbb{G}_{m}\right)^{n}\right) \cong \oplus_{I \subset\{1, \ldots, n\}} \mathbb{Z}\left(\mathbb{G}_{m}^{\wedge|I|}\right)
$$

induces a corresponding splitting of the $\mathbb{A}^{1}$-chain complex $C_{*}^{\mathbb{A}^{1}}\left(\left(\mathbb{G}_{m}\right)^{n}\right)$ as a direct sum of $C_{*}^{\mathbb{A}^{1}}\left(\left(\mathbb{G}_{m}\right)^{\wedge n}\right)$ 's. Consequently, the class of complexes generated by the $\left.\left.C_{*}^{\mathbb{A}^{1}}\left(\left(\mathbb{G}_{m}\right)^{\wedge i}\right)\right\}_{i}\right)$ 's is the same as the one generated by the $\left.\left.C_{*}^{\mathbb{A}^{1}}\left(\left(\mathbb{G}_{m}\right)^{i}\right)\right\}_{i}\right)$ 's.

Definition 4.7. The class of mixed Tate complexes is the class

$$
\mathcal{C}_{\mathbb{A}^{1}}^{\text {Tate }}(k):=\mathcal{C}\left(\left\{C_{*}^{\mathbb{A}^{1}}\left(\left(\mathbb{G}_{m}\right)^{\wedge i}\right)\right\}_{i}\right)=\mathcal{C}\left(\left\{C_{*}^{\mathbb{A}^{1}}\left(\left(\mathbb{G}_{m}\right)^{i}\right)\right\}_{i}\right)
$$

generated by the $\mathbb{A}^{1}$-chain complexes of all the smash-power of $\mathbb{G}_{m}$ 's or equivalently by all the split tori.

The class of strict mixed Tate complexes is the strict class $\mathcal{C}_{\mathbb{A}^{1}}^{\text {str.-Tate }}(k)$ generated by the $\mathbb{A}^{1}$-chain complexes of all the smash-power of $\mathbb{G}_{m}$ 's.

Observe that $\mathcal{C}_{\mathbb{A}^{1}}^{\text {str.-Tate }}(k) \subset \mathcal{C}_{\mathbb{A}^{1}}^{\text {Tate }}(k)$ are both multiplicative and contained in the class of $\mathbb{A}^{1}$-local chain complexes.

4.2. Bruhat decomposition and $\mathbb{A}^{1}$-chain complex of $G$. In this section we deal with:

THEOREM 4.8. Let $G$ be a split semi-simple $k$-group. Then the $\mathbb{A}^{1}$-chain complexes $C_{*}^{\mathbb{A}^{1}}(G)$ of $G$ is of strict mixed Tate type and the $\mathbb{A}^{1}$-chain complex $C_{*}^{\mathbb{A}^{1}}(B G)$ of $B G$ is the suspension of a mixed Tate complex.

If furthermore $G$ is simply connected, then $C_{*}^{\mathbb{A}^{1}}(G)$ is the suspension of a strict mixed Tate complex.

Proof. The first statement follows from the Bruhat decomposition of $G$. Let $T \subset G$ be a split maximal torus, $T \subset B \subset G$ a Borel subgroup containing $T$. There is an increasing filtration of open subsets [55]

$$
\emptyset=\Omega_{s+1} \subset \Omega_{s} \subset \Omega_{s-1} \subset \cdots \subset \Omega_{0}=G
$$

and for each $i$ an isomorphism of schemes

$$
\Omega_{i-1}-\Omega_{i} \cong \amalg_{w \in W \mid \text { length }(w)=i} \mathbb{A}^{i} \times B
$$

where length() is the length function on the Weyl group $W$ of $G$, and $s$ is the maximal possible length. Observe that there is only one element of length $s$ and that the "big cell" $\Omega_{s}$ is an open subscheme isomorphic to $\mathbb{A}^{s} \times B$.

By the theorem of Quillen-Suslin, and its generalization to rings of Laurent polynomials [32, Corollary 4.9 p. 146], the normal bundle of the closed immersion $\Omega_{i-1}-\Omega_{i} \rightarrow \Omega_{i-1}$ is trivial and of rank $s-i$ (remember that as a $k$-variety $B$ is the product of $T$ and an affine space [55].

Now using the homotopy purity theorem of [44], the quotients sheaves $\Omega_{i-1} / \Omega_{i}$ are canonically isomorphic in the $\mathbb{A}^{1}$-homotopy category to the Thom space of the trivial bundle, which means a pointed space of the form 
$S^{s-i} \wedge\left(\mathbb{G}_{m}\right)^{\wedge s-i} \wedge\left(\mathbb{A}^{i} \times B_{+}\right)$. As $T \rightarrow B$ is an $\mathbb{A}^{1}$-homotopy equivalence, it follows that the quotient sheaves $\Omega_{i-1} / \Omega_{i}$ are indeed isomorphic in the pointed $\mathbb{A}^{1}$-homotopy category to $S^{s-i} \wedge\left(\mathbb{G}_{m}\right)^{\wedge s-i} \wedge\left(T_{+}\right)$.

It follows at once that the $\mathbb{A}^{1}$-chain complex $G$ is of mixed Tate type. The statement concerning $C_{*}^{\mathbb{A}^{1}}(B G)$ follows from this, the fact that the class of mixed Tate complexes is multiplicative, by considering the usual skeletal filtration of $B G, *=F^{0}(B G) \subset F^{1}(B G)=S^{1} \wedge G \subset \cdots \subset F^{s}(B G) \subset$ $\ldots B G$, whose quotients are $F^{s}(B G) / F^{s-1}(B G) \cong S^{i} \wedge G^{\wedge i}$.

To prove the last statement, we observe by the Bruhat decomposition that the cone $C$ of $T \subset G$ (do no confound with the homogeneous variety $G / T)$ is always the suspension of a strict mixed Tate type.

Now we saw in Theorem 1.5 that if $G$ is simply connected it is $\mathbb{A}^{1}$ connected. It follows from the results in [41] that the morphism $T \rightarrow G$ is then trivial in the pointed $\mathbb{A}^{1}$-homotopy category. Thus the cone $C$ is isomorphic to the wedge $G \vee \Sigma(T)$ which proves the last property.

REMARK 4.9. Observe that because $B G \rightarrow B \operatorname{Sing} \mathbb{A}^{1}(G)$ is an $\mathbb{A}^{1}$-weak equivalence, it follows that the morphism of chain complexes

$$
C_{*}^{\mathbb{A}^{1}}(B G) \rightarrow C_{*}^{\mathbb{A}^{1}}\left(B \operatorname{Sing} \mathbb{A}^{\mathbb{A}^{1}}(G)\right)
$$

is a quasi-isomorphism.

It follows from all what we have been doing so far, that for $G$ a split semisimple and simply connected algebraic $k$-group, to check that the pointed $\mathbb{A}^{1}$-local simply connected space

$$
\operatorname{BSing}_{\bullet}^{\mathbb{A}^{1}}(G)
$$

has the rigidity property at $\ell$ it suffices to prove that for each $n \geq 1$, the chain complex $C_{*}^{\mathbb{A}^{1}}\left(\left(\mathbb{G}_{m}\right)^{\wedge n} ; \mathbb{Z} / \ell\right)$ has the rigidity property. This follows from Remark 4.6, Theorem 3.9 and Theorem 4.8.

Thus Theorem 3 for $G$ is a consequence of the following Theorem, which by the way, doesn't depend on $G$. It is proven in Section 5 below.

Theorem 4.10. Assume $k$ is a prime field ${ }^{6}$. For any $n \geq 0$ the chain complex

$$
L_{\mathbb{A}^{1}}^{a b} \mathbb{Z}\left(\left(\mathbb{G}_{m}\right)^{\wedge n}\right)=C_{*}^{\mathbb{A}^{1}}\left(\mathbb{G}_{m}^{\wedge n}\right)
$$

has the rigidity property.

REMARK 4.11. We already mentioned that in fact we can prove the strong rigidity property for these complexes. See [43].

\footnotetext{
${ }^{6}$ of characteristic $\neq 2$
} 
Some further questions concerning the $C_{*}^{\mathbb{A}^{1}}\left(\left(\mathbb{G}_{m}\right)^{\wedge n}\right)$ 's. We now mention some concrete consequences of the Theorem 4.10 .

Corollary 4.12. For each $n \geq 1$ the chain complex $C_{*}^{\mathbb{A}^{1}}\left(\left(\mathbb{G}_{m}\right)^{\wedge n} ; \mathbb{Z} / \ell\right)$ is locally constant in the étale topology and thus the canonical morphism between $\mathbb{A}^{1}$-local complexes

$$
C_{*}^{\mathbb{A}^{1}}\left(\left(\mathbb{G}_{m}\right)^{\wedge n} ; \mathbb{Z} / \ell\right) \rightarrow \mathbb{Z} / \ell(n)[n]
$$

is an isomorphism in the étale topology, where the complex $\mathbb{Z} / \ell(n)$ is the mod $\ell$ motivic complex in weight $n$ defined by Suslin-Voevodsky [67].

Proof. Theorem 4.10 implies that $C_{*}^{\mathbb{A}^{1}}\left(\left(\mathbb{G}_{m}\right)^{\wedge n} ; \mathbb{Z}\right)$ and thus $C_{*}^{\mathbb{A}^{1}}$ $\left(\left(\mathbb{G}_{m}\right)^{\wedge n} ; \mathbb{Z} / \ell\right)$ have the rigidity property at $\ell$. This is also true for $\mathbb{Z} / \ell(n)[n]$ by $[67$, Proposition 3.3.3 p. 215]. Thus both complexes are locally constant in the étale topology. Thus it suffices to prove that working over an algebraic closure $F$ of $k$, and given $M$ a constant sheaf of $\mathbb{Z} / \ell$-vector spaces over $S m_{F}$, the morphisms in the derived category of étale sheaves of $\mathbb{Z} / \ell$-vector spaces on $S m_{k}$ to the shifts $M[*]$ from both complexes are equal. The group of morphisms from $C_{*}^{\mathbb{A}^{1}}\left(\left(\mathbb{G}_{m}\right)^{\wedge n} ; \mathbb{Z} / \ell\right)$ to $M[*]$ in the étale topology over $F$ is by definition $\tilde{H}_{e t}^{*}\left(\left(\mathbb{G}_{m}\right)_{F}^{\wedge n} ; M\right)$. By [67, Proposition 3.3 .3 p. 215] the complex $\mathbb{Z} / \ell(n)[n]$ is over $F$ isomorphic to $\left(\mu_{\ell}\right)^{\otimes n}[n]$ in the étale topology. We see that we get the same answer by the usual computation of étale cohomology of $\mathbb{G}_{m}^{n}$.

In fact we conjecture the following. Let $\underline{\mathbf{K}}_{n}^{M W}$ be the sheaf of unramified Milnor-Witt K-theory defined in [41]. There is a canonical epimorphism $\underline{\mathbf{K}}_{n}^{M W} \rightarrow \underline{\mathbf{K}}_{n}^{M}$ and we let $\mathbb{Z}^{\mathbb{A}^{1}}(n)$ be the chain complex of sheaves of abelian groups on $S m_{k}$ in the Nisnevich topology defined as the fiber product

$$
\mathbb{Z}^{\mathbb{A}^{1}}(n):=\mathbb{Z}(n) \times \underline{\mathbf{K}}_{n}^{M}[-n] \underline{\mathbf{K}}_{n}^{M W}[-n]
$$

From $[\mathbf{4 1}]$ there exists a canonical morphism

$$
C_{*}^{\mathbb{A}^{1}}\left(\mathbb{G}_{m}^{\wedge n}\right) \rightarrow \mathbb{Z}^{\mathbb{A}^{1}}(n)[n]
$$

which induces the isomorphism $H_{0}^{\mathbb{A}^{1}}\left(\mathbb{G}_{m}^{\wedge n}\right)=\underline{\mathbf{K}}_{n}^{M W}$ on the 0 homology sheaves.

The following conjecture is a refinement of Corollary 4.12:

Conjecture 4.13. For any field $k$, the morphism

$$
C_{*}^{\mathbb{A}^{1}}\left(\mathbb{G}_{m}^{\wedge n}\right) \rightarrow \mathbb{Z}^{\mathbb{A}^{1}}(n)[n]
$$

is a quasi-isomorphism.

Indeed the fiber of the above morphism is a complex concentrated in degree 0 , equal by [41] and the Milnor conjecture to the sheaf $\underline{I}^{n+1}$ by [37]; now the sheaves $\underline{\mathbf{I}}^{n}$ are zero in the étale topology for $n \geq 1$. 


\section{The rigidity property for the $C_{*}^{\mathbb{A}^{1}}\left(\left(\mathbb{G}_{m}\right)^{\wedge n}\right)^{\prime} \mathbf{s}$}

In this section we prove that the chain complexes $C_{*}^{\mathbb{A}^{1}}\left(\left(\mathbb{G}_{m}\right)^{\wedge n}\right)$ have the rigidity property, completing thus the proof of Theorem 3 and Corollary 6 .

Our method is to endow the homology sheaves of $C_{*}^{\mathbb{A}^{1}}\left(\left(\mathbb{G}_{m}\right)^{\wedge n}\right)$ with a natural structure of sheaf with transfers in a slightly more general (or weaker) sense as the notion of Voevodsky [67]. In Section 5.2 below we achieve the proof of Theorem 3 by showing that this new kind of sheaves have the rigidity property at $\ell$ by reducing to the classical rigidity theorem from [63]. These structure on the homology sheaves are coming in fact from transfers morphisms at the chain complexes level:

$$
R C_{*}^{\mathbb{A}^{1}}\left(\left(\mathbb{G}_{m}\right)^{n}\right)^{L} \rightarrow R C_{*}^{\mathbb{A}^{1}}\left(\left(\mathbb{G}_{m}\right)^{n}\right)^{K}
$$

where $K \subset L$ is a monogenous finite extension between finite type fields extension of $k$, and the notation $R C_{*}^{S}$ denotes the derived internal functional complex. This is done in details in $[\mathbf{4 3}]$.

REMARK 5.1. Contrary to our first belief, the construction of the above transfer morphisms on the $\mathbb{A}^{1}$-localization of the $\mathbb{Z}\left(\left(\mathbb{G}_{m}\right)^{\wedge n}\right)$ are not directly related to the transfers morphisms on the sheaf $\mathbb{G}_{m}^{n}$. It is not so clear to formalize our construction and to write down a minimal list of axioms for a sheaf $M$ that guaranties that the homology sheaves of the chain complex $L_{\mathbb{A}^{1}}^{a b}(\mathbb{Z}(\mathcal{M}))$ will have this type of structure. It seems that a good general candidate for is a sheaf of the form $\mathbb{Z}\left(\mathbb{G}_{m}\right) \otimes M$ where $M$ is any reasonable sheaf. This is still an open question.

On the converse, even if $\mathcal{M}$ be a strictly $\mathbb{A}^{1}$-invariant sheaf with transfers in the sense of Voevodsky then in general the chain complex $L_{\mathbb{A}^{1}}^{a b}(\mathbb{Z}(\mathcal{M}))$ has not the rigidity property at $\ell$. In particular it has no suitable transfers structure in general. For instance take the free sheaf of abelian groups $\mathbb{Z}(E)$ on the sheaf with transfers $\mathcal{M}=E$ given by an elliptic curve $E$ over $k$. Then the sheaf $\mathbb{Z}(E)$ itself is a strictly $\mathbb{A}^{1}$-invariant sheaf, and is thus equal to its own $\mathbb{A}^{1}$-localization. One may check that it has not the rigidity property at $\ell$.

5.1. The Transfers morphisms on the $\mathbb{A}^{1}$-localization of the $\mathbb{Z}\left(\mathbb{G}_{m}^{\wedge n}\right)$ 's. We consider the free pointed sheaf of abelian groups $\mathcal{Z}(n):=$ $\mathbb{Z}\left(\mathbb{G}_{m}^{\wedge n}\right)$ on the smash-product $\mathbb{G}_{m}^{\wedge n}$ (this means that we impose the relation "base point" $=0$ ). We define below an explicit inclusion of sheaves of abelian groups on $S m_{k}$ of the form:

$$
0 \rightarrow \mathcal{Z}(n) \subset \mathcal{Z}_{t r}(n)
$$

with two basic properties:

1) For each monogenous finite extension $K \subset L$ of fields of finite type over $k$, there is a canonical transfer morphism

$$
\mathcal{Z}(n)(L) \rightarrow \mathcal{Z}_{t r}(n)(K)
$$


with nice natural properties; $\mathcal{Z}_{t r}(n)$ is more or less the universal sheaf with this property, it is defined as the sheaf of finite correspondences to $\mathbb{A}^{1}$ with coefficients in $\mathcal{Z}(n)$ in the spririt of [67].

2) The inclusion $\mathcal{Z}(n) \rightarrow \mathcal{Z}_{t r}(n)$ is is an $\mathbb{A}^{1}$-equivalence, in a quite explicit way. In particular applying the $\mathbb{A}^{1}$-localization functor to $\mathcal{Z}(n) \rightarrow \mathcal{Z}_{t r}(n)$ yields a quasi isomorphism of the form:

$$
C_{*}^{\mathbb{A}^{1}}\left(\mathbb{G}_{m}^{\wedge n}\right)=L_{\mathbb{A}^{1}}^{a b}(\mathcal{Z}(n)) \cong L_{\mathbb{A}^{1}}^{a b}(\hat{\mathcal{Z}}(n))
$$

The transfers morphisms from 1 ) and some general fact on $\mathbb{A}^{1}$-localization of derived functional complexes allow one to construct the transfers morphisms on each of the homology sheaves $H_{*}^{\mathbb{A}^{1}}\left(\mathbb{G}_{m}^{\wedge n}\right)$ of $C_{*}^{\mathbb{A}^{1}}\left(\mathbb{G}_{m}^{\wedge n}\right)$ which will endow these homology sheaves with a structure of strictly $\mathbb{A}^{1}$-invariant sheaves with generalized transfers that we describe and use in the next Section 5.2 below, in which we also prove a rigidity theorem.

REMARK 5.2. The transfers morphisms we get are the "correct" ones. For instance the transfers we get in this way on $H_{0}^{\mathbb{A}^{1}}\left(\left(\mathbb{G}_{m}\right)^{\wedge n}\right)$ which is the unramified sheaf of Milnor-Witt K-theory in weight $n, \underline{\mathbf{K}}_{n}^{M W}$ described in [41], are the "usual" ones, and are indeed compatible with both the transfers in Milnor K-theory $\underline{\mathbf{K}}_{n}^{M}$ and with the canonical transfers in the $n$-th power of the fundamental ideal $\underline{\mathbf{I}}^{n}[\mathbf{1}][\mathbf{5 3}]$ through the canonical epimorphisms

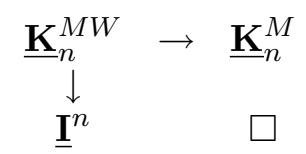

In the sequel, we simply denote the sheaf $\mathcal{Z}(n)$ by $\mathcal{Z}$ when no confusion can arise on $n$, which is fixed everywhere from now on. The sheaf of abelian groups $\mathcal{Z}$ has many good properties. It is an unramified sheaf of abelian groups in the sense of $[\mathbf{4 1}]$, which means that its value on a smooth irreducible $k$-scheme $X$ with function field $K$ is the intersection in $\mathcal{Z}(K)$ of the $\mathcal{Z}\left(\mathcal{O}_{X, x}\right)$ where $x$ runs over the set $X^{(1)}$ of points of codimension 1 in $X$. It is moreover $\mathbb{A}^{1}$-invariant. However $\mathcal{Z}$ is not a strictly $\mathbb{A}^{1}$-invariant sheaf though the sheaf $\mathbb{G}_{m}^{n}$ has this property; this is the whole point of the story of course!

We start by observing that the sheaf $\mathcal{Z}$ admits a canonical structure of $\mathbb{Z}\left[\mathbb{G}_{m}\right]$-module. Here $\mathbb{Z}\left[\mathbb{G}_{m}\right]$ is the free sheaf of abelian groups on the sheaf of groups $\mathbb{G}_{m}$ (no condition on the base point) and as such is indeed a sheaf of commutative rings. We take the multiplication

$$
\begin{gathered}
\mathbb{Z}\left[\mathbb{G}_{m}\right] \times \mathbb{Z}\left(\left(\mathbb{G}_{m}\right)^{\wedge n}\right) \rightarrow \mathbb{Z}\left(\left(\mathbb{G}_{m}\right)^{\wedge n}\right) \\
\left(U,\left\{\alpha_{1}, \ldots, \alpha_{n}\right\}\right) \rightarrow\left\{U \alpha_{1}, \alpha_{2}, \ldots, \alpha_{n}\right\}-\left\{U, \alpha_{2} \ldots, \alpha_{n}\right\}
\end{gathered}
$$

(the correcting term is there to garanty that the map factors through the smash product.) 
For any integer $n \in \mathbb{N}$, we set $[n]_{\epsilon}=\sum_{i=0}^{n-1}\left\{(-1)^{i}\right\}$ which we consider as an element of $\mathbb{Z}\left[\mathbb{G}_{m}\right](k)=\mathbb{Z}\left[k^{\times}\right]$. Observe that one has the easy formula

$$
[n]_{\epsilon} \cdot[m]_{\epsilon}=[n m]_{\epsilon}
$$

The above $\mathbb{Z}\left[\mathbb{G}_{m}\right]$-structure will a priori play a little role in the constructions below, only through the action of the $[n]_{\epsilon}$. However, to check the property of the transfers morphims it will be essential. See Section 5.2 below.

If $X$ is a smooth $k$-scheme we let $\mathcal{C}\left(X ; \mathbb{A}^{1}\right)$ be the set of points $y \in \mathbb{A}^{1} \times X$ such that the closure $\bar{y}$ (an irreducible closed integral subscheme in $\mathbb{A}^{1} \times X$ ) is finite over $X$ and dominate an irreducible component of $X$ (see [67]). We now define the sheaf $\mathcal{Z}_{t r}$. Its values on a smooth $k$-scheme $X$ is the abelian group:

$$
\mathcal{Z}_{t r}(X):=\oplus_{y \in \mathcal{C}\left(X, \mathbb{A}^{1}\right)} \mathcal{Z}(\bar{y})
$$

One of the hidden points of this definition is that we may actually define $\mathcal{Z}(\bar{y})$, which means that $\mathcal{Z}$ as a presheaf can be extended to singular $k$ schemes of the type $\bar{y}$.

Observe also that if $X$ is irreducible with function field $K$ one has a canonical inclusion $\mathcal{Z}_{t r}(X) \subset \mathcal{Z}_{t r}(K)$. This follows from the fact that $Y \times_{X}$ $\operatorname{Spec}(K) \rightarrow Y$ induces an injection $\mathcal{Z}(Y) \subset \mathcal{Z}\left(Y \times_{X} \operatorname{Spec}(K)\right)$.

Now we turn this definition into a presheaf on $S m_{k}$ as follows. Let $f: X^{\prime} \rightarrow X$ be a morphism in $S m_{k}$. We want to define $\mathcal{Z}_{t r}(f): \mathcal{Z}_{t r}(X) \rightarrow$ $\mathcal{Z}\left(X^{\prime}\right)$. Clearly if $X=X_{1} \amalg X_{2}$ the group $\mathcal{Z}_{t r}(X)$ splits accordingly as $\mathcal{Z}_{t r}\left(X_{1}\right) \oplus \mathcal{Z}_{t r}\left(X_{2}\right)$. To define $\mathcal{Z}_{t r}(f)$ we may thus assume that $X^{\prime}$ and $X$ are irreducible.

Assume first that $f$ is dominant. Let $K$ be the function field of $X$ and $L$ that of $X^{\prime}$. As $\mathcal{Z}_{t r}(X) \subset \mathcal{Z}_{t r}(K)$ and $\mathcal{Z}_{t r}\left(X^{\prime}\right) \subset \mathcal{Z}_{t r}(L)$ we need first to define the morphism $\mathcal{Z}_{t r}(K) \rightarrow \mathcal{Z}_{t r}(L)$ induced by the field extension $\phi: K \subset L$.

Given $y \in \mathcal{C}\left(\operatorname{Spec}(K) ; \mathbb{A}^{1}\right)=\left(\mathbb{A}_{K}^{1}\right)^{(1)}$, we denote by $\{z \mid y\}$ the finite set of closed points in $\mathbb{A}_{L}^{1}$ which map to $y$ through the induced morphism $\mathbb{A}_{L}^{1} \rightarrow \mathbb{A}_{K}^{1}$ and we write $z \mid y$ to mean $z \in\{z \mid y\}$. In other words, the set of such $z$ is exactly the set of points in the closed subscheme $\operatorname{Spec}\left(\kappa(y) \otimes_{E} F\right)$ of $\mathbb{A}_{F}^{1}$. For each $z \mid y$ we let $\lambda(z \mid y) \in \mathbb{N}$ denote the lenght of $z$ in $\kappa(y) \otimes_{E} F$. Then we take for morphism $\mathcal{Z}_{t r}(K) \rightarrow \mathcal{Z}_{t r}(L)$ the direct sum indexed by the $y$ 's of the following homomorphism whose source is the factor $\mathcal{Z}(\kappa(y))$ of $\mathcal{Z}_{t r}(K)$ and the target the summand $\oplus_{z \mid y} \mathcal{Z}(\kappa(z))$ of $\mathcal{Z}_{t r}(L)$ :

$$
\sum_{z \mid y}[\lambda(z \mid y)]_{\epsilon} \cdot \mathcal{Z}\left(\phi_{z}\right): \mathcal{Z}(\kappa(y)) \rightarrow \oplus_{z \mid y} \mathcal{Z}(\kappa(z))
$$

Here $\phi_{z}$ is the induced fields extension $\kappa(y) \subset \kappa(z)$ and $\mathcal{Z}\left(\phi_{z}\right): \mathcal{Z}(\kappa(y)) \rightarrow$ $\mathcal{Z}(\kappa(z))$ the induced group homomorphism.

One checks that this construction induces a functor $\left(\tilde{S m_{k}}\right)^{o p} \rightarrow \mathcal{A} b$ from smooth $k$-schemes and dominant morphisms to that of abelian groups. It 
remains to extends this to a functor $\left(S m_{k}\right)^{o p} \rightarrow \mathcal{A} b$ and for this we first define restriction morphisms

$$
\mathcal{Z}_{t r}(X) \rightarrow \mathcal{Z}_{t r}(Z)
$$

for any closed immersion $Z \subset X$ in $S m_{k}$ everywhere of codimension 1 .

One may reduce to $X$ being the localization of the generic point of $Z$, that is to say $X=\operatorname{Spec}(A)$ where $A$ is a geometric discrete valuation ring with residue field $\kappa$ the function field of $Z$. In that case given $y \in \mathcal{C}\left(X, \mathbb{A}^{1}\right)$ we let $\mu_{y}$ be the length of the finite $\kappa$-scheme $\bar{y} \times_{X} \operatorname{Spec}(\kappa)$. Let also $t(y) \in$ $\mathcal{C}\left(\operatorname{Spec}(\kappa), \mathbb{A}^{1}\right)$ the image of $y$ in $\mathbb{A}_{\kappa}^{1}$. We take for morphism

$$
\mathcal{Z}_{t r}(X)=\mathcal{Z}_{t r}(\operatorname{Spec}(A)) \rightarrow \mathcal{Z}_{t r}(\operatorname{Spec}(\kappa))=\mathcal{Z}_{t r}(Z)
$$

the direct sum over the $y$ 's of the morphisms of the form:

$$
[\mu]_{\epsilon}: \mathcal{Z}(\bar{y}) \rightarrow \mathcal{Z}(\kappa(t(y)))
$$

induced by the restriction morphisms on $\mathcal{Z}$. One may check:

THEOREM 5.3. The previous construction extends uniquely to a presheaf of $\mathbb{Z}\left[\mathbb{G}_{m}\right]$-modules $\mathcal{Z}_{t r}$ on $S m_{k}$. This presheaf is a sheaf in the Nisnevich topology. Moreover the natural transformation $\mathcal{Z} \rightarrow \mathcal{Z}_{\text {tr }}$ which corresponds to the zero section $0 \in \mathcal{C}\left(-, \mathbb{A}^{1}\right)$ is a monomorphism of sheaves (of $\mathbb{Z}\left[\mathbb{G}_{m}\right]$ modules).

Now we prove that the inclusion $\mathcal{Z} \rightarrow \mathcal{Z}_{t r}$ is an $\mathbb{A}^{1}$-equivalence. The proof is completely explicit and proceeds in several steps (basically 3 ). First we observe that $\mathcal{Z}_{t r}$ contains a canonical subsheaf which we denote by $c\left(-; \mathbb{A}^{1}\right) \tilde{\otimes} \mathcal{Z}$ whose values on the henselization $A$ of a point in a smooth $k$-scheme is equal to

$$
c\left(\operatorname{Spec}(A) ; \mathbb{A}^{1}\right) \otimes \mathcal{Z}(A)=\oplus_{y \in \mathcal{C}\left(\operatorname{Spec}(A), \mathbb{A}^{1}\right)} \mathcal{Z}(A)
$$

It is straithforward to check that these form a subsheaf of $\mathcal{Z}_{t r}$. The notation $c\left(-; \mathbb{A}^{1}\right) \tilde{\otimes} \mathcal{Z}$ means that the sheaf is not exactly the tensor product, as the restriction morphisms use the multiplication by the $[n]_{\epsilon}$ 's at some point.

Now the obvious $\mathbb{A}^{1}$-homotopy on $c\left(-, \mathbb{A}^{1}\right)$ "multilplication by the scalar" which gives an $\mathbb{A}^{1}$-deformation of $c\left(-, \mathbb{A}^{1}\right)$ onto the constant sheaf $\mathbb{Z}$ extends to induce an $\mathbb{A}^{1}$-deformation of $c\left(-; \mathbb{A}^{1}\right) \tilde{\otimes} \mathcal{Z}$ onto $\mathcal{Z}$. Thus, using the factorization $\mathcal{Z} \subset c\left(-; \mathbb{A}^{1}\right) \tilde{\otimes} \mathcal{Z} \subset \mathcal{Z}_{t r}$ of the inclusion $\mathcal{Z} \subset \mathcal{Z}_{t r}$, we see that it suffices to prove that the last inclusion $c\left(-; \mathbb{A}^{1}\right) \tilde{\otimes} \mathcal{Z} \subset \mathcal{Z}_{t r}$ is an $\mathbb{A}^{1}$ equivalence, or in other words that the cokernel $\overline{\mathcal{Z}}_{t r}$ of the latter inclusion is $\mathbb{A}^{1}$-equivalent to 0 .

To prove that $\overline{\mathcal{Z}}_{t r}$ is $\mathbb{A}^{1}$-trivial we will embedd it into an other sheaf of abelian groups $\overline{\mathcal{Z}}$ and we will show that both $\overline{\mathcal{Z}}_{\text {tr }}$ and the cokernel $\hat{\mathcal{Z}}:=$ $\overline{\mathcal{Z}} / \overline{\mathcal{Z}}_{\text {tr }}$ admit an explicit $\mathbb{A}^{1}$-homotopy to zero.

Now the cokernel $\overline{\mathcal{Z}}_{t r}$ has the following values on the henselization $A$ of a point in a smooth $k$-scheme

$$
\overline{\mathcal{Z}}_{t r}(A)=\oplus_{y \in \mathcal{C}\left(\operatorname{Spec}(A), \mathbb{A}^{1}\right)}(\mathcal{Z}(\bar{y}) / \mathcal{Z}(A))
$$


where $\mathcal{Z}(\bar{y}) / \mathcal{Z}(A)$ is the cokernel of the injective morphism

$$
\mathcal{Z}(A) \subset \mathcal{Z}(\bar{y})
$$

The sheaf $\overline{\mathcal{Z}}$ will have values on $\operatorname{Spec}(A)$ the group

$$
\overline{\mathcal{Z}}(A)=\oplus_{x \in\left(\mathbb{A}_{F}^{1}\right)^{(1)}}(\mathcal{Z}(A[x]) / \mathcal{Z}(A))
$$

where $F$ is the fraction field of $A$, and for each closed point $x$ of $\mathbb{A}_{F}^{1}$ we let $A[x] \subset \kappa(x)$ be the sub- $A$-algebra of the residue field $\kappa(x)$ of $x$ generated by $x$ itself, viewed as a function $\operatorname{Spec}(\kappa(x)) \rightarrow \mathbb{A}_{F}^{1}$. We will write in the sequel $\mathcal{Z}_{0}(A[x])$ for $\mathcal{Z}(A[x]) / \mathcal{Z}(A)$.

Observe that though $\kappa(x)$ is a finite extension of $F$, in general $x$ is not integral over $A$ and $A[x]$ is not a finite type $\mathbb{A}$-module. The $x$ which have this property are exactly the one integral over $A$ which corresponds exactly to the $y \in \mathcal{C}\left(\operatorname{Spec}(A), \mathbb{A}^{1}\right)$ and gives the inclusion

$$
\overline{\mathcal{Z}}_{t r} \subset \overline{\mathcal{Z}}
$$

The only problem to construct $\overline{\mathcal{Z}}$ as a sheaf is to define the reduction morphisms

$$
\overline{\mathcal{Z}}(A) \rightarrow \overline{\mathcal{Z}}(B)
$$

where $A \rightarrow B$ is the morphism corresponding to a closed essentially smooth $k$-scubscheme $\operatorname{Spec}(B) \subset \operatorname{Spec}(A)$. The basic idea is simple. Take an $x$ as above. The kernel of the epimorphism $A[X] \rightarrow A[x], X \mapsto x$ is of course generated by a primitive Polynomial $P_{0}=a_{n} X^{n}+a_{n-1} X^{n-1}+\cdots+a_{0} \in A[X]$ such that $\frac{1}{a_{n}} P_{0} \in F[X]$ is the minimal polynomial $P$ of $x$ over $F$. Given an epimorphism $A \rightarrow B$ as above, one wants to define the reduction morphism on the summand $\mathcal{Z}_{0}(A[x])$ by using the epimorphism from the algebra $A[X] /\left(P_{0}\right) \cong A[x]$ onto $B[X] /\left(\bar{P}_{0}\right)$, where $\bar{P}_{0} \in B[X]$ is the reduction of $P_{0}$. We proceed as follows. If $\bar{P}_{0} \neq 0 \in B[X]$, as $B$ is also a UFD, one may write

$$
\bar{P}_{0}=c \Pi_{i} Q_{i}^{n_{i}}
$$

for some primitive irreducible polynomials $Q_{i}$ of $B[X]$, and $c \in B-\{0\}$. We write $x_{i}$ for the closed point in $\mathbb{A}_{K}^{1}$ corresponding to $Q_{i}$, where $K$ is the fraction field of $B$. For each $i$ we have a canonical epimorphism of $A$-algebras

$$
A[x] \rightarrow B\left[x_{i}\right]
$$

We then define the reduction morphism on the summand $\mathcal{Z}_{0}(A[x])$ as the sum

$$
\Sigma_{i}\left[n_{i}\right]_{\epsilon} \mathcal{Z}_{0}\left(A[x] \rightarrow B\left[x_{i}\right]\right): \mathcal{Z}_{0}(A[x]) \rightarrow \oplus_{i} \mathcal{Z}_{0}\left(B\left[x_{i}\right]\right)
$$

If $\bar{P}_{0}=0 \in B[X]$, we just take 0 for the reduction morphism on the summand $\mathcal{Z}_{0}(A[x])$.

Lemma 5.4. The previous definition can be extended in a unique way to a sheaf of abelian groups in the Nisnevich topology $\left(S m_{k}\right)^{o p} \ni X \mapsto \overline{\mathcal{Z}}(X)$. 
The proof is straightwforward. This construction works only because we work with the sum of the $\mathcal{Z}_{0}(A[x])$. There is no way to define a sheaf structure whose values on $A$ would be $\oplus_{x \in\left(\mathbb{A}_{F}^{1}\right)^{(1)}} \mathcal{Z}(A[x])$. The main obstruction is when the reduction of $P_{0}$ in $B[X]$ vanishes (see above) one doesn't know where to send $\mathcal{Z}(A[x])$. Killing the $\mathcal{Z}(A)$ 's everywhere kills precisely this obstruction; for the details see $[\mathbf{4 3}]$.

Now it remains to explicit on both $\overline{\mathcal{Z}}$ and the quotient $\hat{\mathcal{Z}}=\overline{\mathcal{Z}} / \overline{\mathcal{Z}}_{\text {tr }}$ an $\mathbb{A}^{1}$ homotopy to zero. For the first one we proceed as follows. For $F$ a field extension of $k$, we have the obvious action of $F$ on $\overline{\mathcal{Z}}(F)=\oplus_{x \in\left(\mathbb{A}_{F}^{1}\right)^{(1)}} \mathcal{Z}_{0}(\kappa(x))$ which let 0 acts by the zero morphism and which let $f \in F^{\times}$acts by permuting the $x$ 's by $x \mapsto \frac{x}{f}$.

LEMMA 5.5. The previous construction extends uniquely to a morphism of sheaves $\mathbb{A}^{1} \times \overline{\mathcal{Z}} \rightarrow \overline{\mathcal{Z}}$ which defines an $\mathbb{A}^{1}$-homotopy between the Identity morphism and the zero morphism.

Proof. One has basically to check that given an henselization $A$ of a point in a smooth $k$-scheme the multiplication by $f: \overline{\mathcal{Z}}(F) \rightarrow \overline{\mathcal{Z}}(F)$ preserves $\overline{\mathcal{Z}}(A)$ and induces $f: \overline{\mathcal{Z}}(A) \rightarrow \overline{\mathcal{Z}}(A)$ and that moreover given an epimorphism $A \rightarrow B$ as above, that the obvious diagram

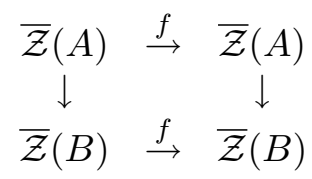

commutes. To check this one may reduce to the case $\operatorname{Spec}(B) \subset \operatorname{Spec}(A)$ is of codimension one, and then by localization also to the case $B$ is a field. This now can be hand checked: if $f$ is a unit it is clear, if not we may reduce to the case where $f$ is an irreducible element of $A$, that is to say when $f$ generates the maximal ideal of $A$. But then given a point $x$, in the sequence $f^{i} \cdot x$ of points (related by the multiplication by $f$ ), there exists one and only one $i \in \mathbb{Z}$ such that $f^{i} \cdot x \in \kappa(x)$ is integral over $A$ and $f^{i-1} . x$ is not. In that case each summand $\mathcal{Z}_{0}\left(A\left[f^{j} . x\right]\right), j \neq i$, will reduce to 0 in $\mathcal{Z}_{0}(B)$ (as the point $f^{j} . x$ specializes to 0 for $j>i$ and to $\infty$ for $j<i$ ). This implies clearly the claim as both composition morphisms in the diagram are then zero.

We know claim that the sheaf $\hat{\mathcal{Z}}$ is aslo $\mathbb{A}^{1}$-contractible by an explicit $\mathbb{A}^{1}$-homotopy to 0 . More precisely for $A$ the henselization of a point in a smooth $k$-scheme, with field of fractions $F$ and residue field $\kappa$, we have

$$
\hat{\mathcal{Z}}(A)=\oplus_{x \in\left(\mathbb{A}_{F}^{1}\right)^{(1)} \mid x_{\infty} \neq \emptyset} \mathcal{Z}_{0}(A(x))
$$

where we mean $x_{\infty} \neq \emptyset$ if and only if $\bar{P}_{0}$ is constant in $\kappa[X]$ (observe that this holds exactly when the closure $\bar{x} \subset \mathbb{P}_{A}^{1}$ of the point $x$ meets the section at infinity $\left.\operatorname{Spec}(A) \subset \mathbb{P}_{A}^{1}\right)$. 
We take the action of $A$ on $\hat{\mathcal{Z}}(A)$ which let 0 acts by the zero morphism and which let $f \in A-\{0\}$ acts by mapping (again) $x$ to $\frac{x}{f}$. This defines an $\mathbb{A}^{1}$-homotopy of the identity of $\hat{\mathcal{Z}}$ to 0 and proves the claim.

REMARK 5.6. Contrary to the appearances, this homotopy, though very close to the previous one, is not compatible with the one on $\overline{\mathcal{Z}}$, and the epimorphism $\overline{\mathcal{Z}} \rightarrow \hat{\mathcal{Z}}$ doesn't preserve the $\mathbb{A}^{1}$-homotopy. In fact most probably, there is no $\mathbb{A}^{1}$-homotopy of the identity morphism of $\overline{\mathcal{Z}}_{t r}$ to 0 .

5.2. Transfers structures on the $H_{*}^{\mathbb{A}^{1}}\left(\left(\mathbb{G}_{m}\right)^{n}\right)$ 's and rigidity. We now write down explicitely the structures that we get on the homology sheaves $H_{*}^{\mathbb{A}^{1}}\left(\left(\mathbb{G}_{m}\right)^{n}\right)$ 's by the construction of the previous section.

First, the $\mathbb{Z}\left[\mathbb{G}_{m}\right]$-module structure can be seen to induce a structure of $b G W$-modules on these. Recall that $\mathbf{G W}$ denotes the sheaf of unramified Grothendieck-Witt groups on $S m_{k}\left[\overline{\mathbf{4 1}]}\right.$. This is the strictly $\mathbb{A}^{1}$-invariant sheaf of unramified Milnor-Witt K-theory $\underline{\mathbf{K}}_{0}^{M W}$ in weight 0 constructed in [41]. This is a sheaf of commutative rings and its sections on a field $F \in \mathcal{F}_{k}$ is the ring of non-degenerated symmetric bilinear forms over $F$ defined and studied in [36]. We denote as usual by $\langle a\rangle \in G W(F)$ the form of rank 1 associated to a unit $a \in F^{\times}$. There is an induced rank

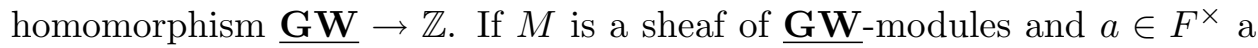
unit, $\langle a>: M(F) \rightarrow M(F), \mu \mapsto<a>\mu$ is thus the multiplication by the class $\langle a\rangle \in G W(F)$.

For any integer $n \in \mathbb{N}$, we set

$$
[n]_{\epsilon}=\sum_{i=0}^{n-1}<(-1)^{i}>\in G W(\kappa)
$$

If $n=2 m$ is even then $[n]_{\epsilon}=m . h$ with $h=1+<-1>$ being the hyperbolic plane. If $n=2 m+1$ is odd, then $[n]_{\epsilon}=1+m . h$.

We will need the following notion ${ }^{7}$ in the next definition. Given a field $K$ of characteristic $p$ and a unitary irreducible polynomial $P \in K[X]$ we may write canonically $P$ as $P_{0}\left(X^{p^{m}}\right)$ where $P_{0}$ is a unitary irreducible separable polynomial and $m \in \mathbb{N}$ an integer. This corresponds to the canonical factorization of $K \subset L=k[X] / P$ as $K \subset L_{\text {sep }} \subset L$ where $K \subset L_{\text {sep }}$ is separable and $L_{\text {sep }} \subset L$ purely inseparable. $m$ is thus the smallest integer with $x^{p^{m}} \in L_{\text {sep }}$ and $\left[L: L_{s e p}\right]=p^{m}$. $P_{0}$ is the minimal polynomial of $x^{p^{m}}$ over $K$. We will denote by $P_{0}^{\prime}$ the derivative of $P_{0}$; thus $P_{0}^{\prime} \neq 0$.

If $\operatorname{char}(K)=0$ then we just take $P=P_{0}$ and $m=0$. Observe that if one has a root $x$ of $P$ in an extension $K \subset L$ then $P_{0}^{\prime}(x) \in L^{\times}$in any characteristic.

Definition 5.7. Let $M$ be a sheaf of $\mathbf{G W}$-modules. A structure of generalized transfers on $M$ consists of the following Datum:

\footnotetext{
${ }^{7}$ The reader who doesn't want to care about inseparability problem in finite characteristic might ignore these and asume everywhere that the fields (or rings) are perfect
} 
(D) For each finite extension $\phi: K \subset L$ in $\mathcal{F}_{k}$ a morphism:

$$
\operatorname{Tr}(\phi)=\operatorname{Tr}_{K}^{L}: M(L) \rightarrow M(K)
$$

called the transfer morphism from $L$ to $K$.

This Datum should satisfy the following 4 axioms:

(A0) Functoriality. $T r_{K}^{K}=I d_{M(K)}: M(K) \rightarrow M(K)$ and for any finite extensions $K \subset F \subset L$ in $\mathcal{F}_{k}$ then one has $\operatorname{Tr}_{K}^{F} \circ \operatorname{Tr}_{F}^{L}=\operatorname{Tr}_{K}^{L}$ : $M(L) \rightarrow M(K)$.

(A1) Projection Formulas. Let $\phi: K \subset L$ in $\mathcal{F}_{k}$ be a finite extension. For $a \in K^{\times}$and $\mu \in M(L)$ one has $\operatorname{Tr}_{K}^{L}(<\phi(a)>\mu)=<a>\operatorname{Tr}_{K}^{L}(\mu) \in$ $M(K)$. In the same way, if $a \in L^{\times}$and $\mu \in M(F)$ then one has $\operatorname{Tr}_{K}^{L}(<$ $a>. \phi(\mu))=\operatorname{Tr}_{K}^{L}(<a>) . \mu \in M(K)$, where $\operatorname{Tr}_{K}^{L}(<a>) \in G W(K)$ is the usual transfer from $\langle a\rangle$.

(A2) Let $\phi: K \subset L$ be a finite extension in $\mathcal{F}_{k}$ and $\psi: K \subset E$ be an extension in $\mathcal{F}_{k}$. Let $R$ be the tensor product $E \otimes_{K} L$, a finite $E$-algebra. For $x \in \operatorname{Spec}(R)$ let $\phi_{x}: E \subset \kappa(x)$ and $\psi_{x}: L \subset \kappa(x)$ be the corresponding extensions in $\mathcal{F}_{k}$, with $\phi_{x}: E \subset \kappa(x)$ finite. Let $\lambda_{x}$ be the length of the local ring $R_{x}$. Then

$$
M(\psi) \circ \operatorname{Tr}_{K}^{L}=\sum_{x \in \operatorname{Spec}(R)}\left[\lambda_{x}\right]_{\epsilon} \operatorname{Tr}_{E}^{\kappa(x)} \circ M\left(\psi_{x}\right)
$$

(A3) Let $\phi: K \subset L$ be a finite extension in $\mathcal{F}_{k}$, let $A \subset K$ be a geometric discrete valuation ring of $K$ with residue field $\kappa$. We assume that the integral closure $B$ of $A$ in $L$ is also a discrete valuation ring with residue field $\lambda$. Let $\kappa \subset \lambda$ be the induced residue fields extension and let $e \in \mathbb{N}$ be the ramification index. We let $s_{B}: M(B) \rightarrow M(\lambda)$ and $s_{A}: M(A) \rightarrow M(\kappa)$ be the restriction maps.

(A3)(1) If $e=1$ that is to say $[\lambda: \kappa]=[L: K]$, then $\operatorname{Tr}_{K}^{L}$ maps $M(B) \subset M(L)$ into $M(A) \subset M(K)$. Denote by $\operatorname{Tr}_{A}^{B}: M(B) \rightarrow M(A)$ the induced morphism. Then moreover the following diagram commutes

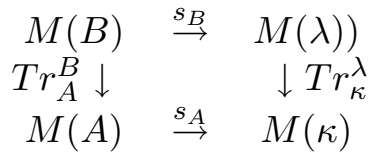

(A3)(2) If $\operatorname{Spec}(B) \rightarrow \operatorname{Spec}(A)$ is monogenous, let $x \in B$ be a generator of $B$ over $A$ and let $P$ be its minimal polynomial. Let $Q \in \kappa[X]$ be the minimal polynomial of the image $\bar{x}$ of $x$ in $\lambda$; observe that $\bar{x}$ is a generator of $\lambda$ over $\kappa$ and that the $\bmod \kappa$ reduction $\bar{P}$ of $P$ is equal to $Q^{e}$. Then the composition $M(L) \stackrel{<P_{0}^{\prime}(x)>}{\longrightarrow} M(L) \stackrel{\operatorname{Tr}_{K}^{L}}{\rightarrow} M(K)$ maps $M(B)$ in $M(A)$. Let $\tau_{A}^{B}(x): M(B) \rightarrow M(A)$ be the induced morphism and denote $\tau_{\kappa}^{\lambda}(\bar{x})$ the composition $M(\lambda) \stackrel{<Q_{0}^{\prime}(x)>}{\longrightarrow} M(\lambda) \stackrel{\operatorname{Tr}_{K}^{L}}{\longrightarrow} M(\kappa)$. Then moreover the following 
diagram commutes

$$
\begin{array}{cccc}
M(B) & \stackrel{s_{B}}{\rightarrow} & M(\lambda) \\
\tau_{A}^{B}(x) \downarrow & & \downarrow & {[e]_{\epsilon} \tau_{\kappa}^{\lambda}(\bar{x})} \\
M(A) & \stackrel{s_{A}}{\rightarrow} & M(\kappa)
\end{array}
$$

EXAMPLE 5.8. For a given integer $n \in \mathbb{Z}$ the sheaves $\underline{\mathbf{K}}_{n}^{M W}$ may be shown to have such a structure, in a canonical way. The transfers morphisms are compatible with the one in Milnor $K$-theory through the projection $\underline{\mathbf{K}}_{n}^{M W} \rightarrow \underline{\mathbf{K}}_{n}^{M}$ and to the canonical transfers in $\underline{\mathbf{I}}^{n}$ (see [53]) through the projection $\underline{\mathbf{K}}_{n}^{M W} \rightarrow \underline{\mathbf{I}}^{n}$.

REMARK 5.9. An $\mathbb{A}^{1}$-invariant sheaf with transfers $M$ in the sense of Voevodsky defines such a structure of generalized transfers, in which the

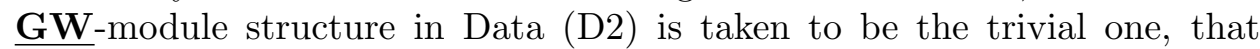
is to say $\mathbf{G W}$ acts through the rank $\underline{\mathbf{G W}} \rightarrow \mathbb{Z}$, and in which the Data (D1) comes by evaluating the transfers on the fields. We will see below in Theorem 5.16 that the converse holds.

REMARK 5.10. For any $\mathbb{P}^{1}$ (or $T$ ) spectrum $E$ the homotopy sheaves $\pi_{n}^{\mathbb{A}^{1}}(E)$ do have this structure. In fact a more careful (and non trivial) analysis shows that for any strictly $\mathbb{A}^{1}$-invariant sheaf $M$ the sheaf $M_{-1}$ admits a canonical structure of generalized transfers. It is still an open question to kow if the converse holds. Is it true that any strictly $\mathbb{A}^{1}$-invariant sheaf with generalized transfers is of the form $M_{-1}$ ?

Remark 5.11. From Axiom (A3)(1) it follows that in particular any finite étale morphism $Y \rightarrow X$ between smooth $k$-schemes the morphism $\operatorname{Tr}_{K}^{L}: M(L) \rightarrow M(K)$ induces a canonical transfer morphism $M(B) \rightarrow$ $M(A)$.

Note that the two parts of Axiom (A3) are not independant. For instance $(\mathbf{A 3})(\mathbf{2})$ in the case $e=1$ implies $(\mathbf{A 3})(\mathbf{1})$ for a monogenous extension $A \subset B$. An other particular case of Axiom (A3)(2) is when $\operatorname{Spec}(B) \rightarrow \operatorname{Spec}(A)$ is totally ramified that is to say $e=[L: K]$ and $\kappa \subset \lambda$ is an isomorphism.

In characteristic 0 the two previous cases $(e=1$ and $e=[L: K])$ are sufficient to characterize the generalized transfer structure. In finite characteristic it is a bit more tricky.

For a more conceptual approach on these Axioms involving modules of Kähler differential forms see $[\mathbf{4 1}, \mathbf{4 3}]$. We gave the previous list of axioms, which doesn't seem "conceptual" because it is exactly the way we are going to check the properties by hands on the $\mathbb{A}^{1}$-complexes of tori in $[\mathbf{4 3}]$. It would be interesting to have a more conceptual proof of these facts. 
Here is for us the most interesting example:

THEOREM 5.12. Let $n$ be an integer. Let $k$ be a perfect field. The $\mathbb{Z}\left[\mathbb{G}_{m}\right]$ module structure and the transfers morphisms induced on the sheaves

$$
H_{*}^{\mathbb{A}^{1}}\left(\left(\mathbb{G}_{m}\right)^{\wedge n}\right)=H_{*}\left(L _ { * } ^ { \mathbb { A } ^ { 1 } } \left(\mathbb{Z}\left(\left(\mathbb{G}_{m}\right)^{\wedge n}\right)\right.\right.
$$

by the constructions of the previous section define a structure of strictly $\mathbb{A}^{1}$-invariant sheaves with generalized transfers in the previous sense.

The proof is straightforward though quite lenghty $[43]$.

The rigidity Theorem. We now state and prove a rigidity theorem for strictly $\mathbb{A}^{1}$-invariant sheaves with generalized transfers. The proof reduces to the "classical" rigidity Theorem of $[\mathbf{6 3}]$.

TheOREM 5.13. Let $M$ be a strictly $\mathbb{A}^{1}$-invariant sheaf of abelian groups with generalized transfers. Then $M$ satisfies the rigidity property at $\ell$.

Proof of Theorem 3. From Theorem 5.12 and the previous Theorem, applied to the field $k$ being a prime field, we get Theorem 4.10 which we already know implies Theorem 3 as well.

Let us mention a stronger form of Theorem 5.13 which is not really needed as far as the Friedlander-Milnor conjecture is concerned:

Theorem 5.14. Assume that if $\ell=2$ then any field $F \in \mathcal{F}_{k}$ is of finite virtual cohomological 2-dimension. Let $M$ be a strictly $\mathbb{A}^{1}$-invariant sheaf of abelian groups with generalized transfers. Then $M$ satisfies the strong rigidity property at $\ell$.

For the case $\ell \neq 2$, in fact the proof of 5.13 follows directly from the classical rigidity theorem [63] and the following observation (observe that to prove Theorem 5.13 we may always assume that -1 is a square):

Lemma 5.15. Assume -1 is a square in $k$. Let $M$ be a strictly $\mathbb{A}^{1}$ invariant sheaf of $\mathbb{Z}\left[\frac{1}{2}\right]$-modules with generalized transfers. Then the $\mathbf{G} \mathbf{W}$ module structure is trivial and thus $M$ is in fact a sheaf with transfers in the sense of Voevodsky by Theorem 5.16 below.

Proof. By Theorem 5.16 it suffices to prove that the $\mathbf{G W}$-module structure is trivial. But as -1 is a square, $1+<-1>=2$ is 0 in $I(k)$. The sheaf $\underline{I}$ is thus a sheaf of $\mathbb{Z} / 2$-modules, which automatically acts trivially on a $\mathbb{Z}\left[\frac{1}{2}\right]$-module. Thus the $\mathbf{G W}$-module structure is indeed trivial.

The following establishes a converse to Remark 5.9.

THEOREM 5.16. Let $M$ be a strictly $\mathbb{A}^{1}$-invariant sheaf of abelian groups with a structure of generalized transfers. Assume that the structure of $\mathbf{G W}$ modules is trivial in the previous sense. Then there exists a unique structure of sheaf with transfers on $M$ in the sense of Voevodsky which induces the structure of generalized transfers. 
To treat the case $\ell=2$ (as well as the more general Theorem 5.14) we will have to work a bit more. More precisely we observe:

TheOREM 5.17. [43] Let $M$ be a strictly $\mathbb{A}^{1}$-invariant sheaf $M$ with generalized transfers. There exists a unique sub-sheaf $\mathcal{I} M \subset M$ which has the following properties:

1) the group of sections $\mathcal{I} M(F)$ on a field $F \in \mathcal{F}_{k}$ is the sub-GW(F)module generated by the images $\operatorname{Tr}_{K}^{L}(I(L) \cdot M(L))$ of the $I(L) \cdot M(L)$ through the transfer morphism $\operatorname{Tr}_{K}^{L}: M(L) \rightarrow M(K)$ where $L$ runs over each finite extensions of $K$;

2) $\mathcal{I} M$ is strictly $\mathbb{A}^{1}$-invariant;

Moreover, $\mathcal{I} M$ is stable both by the structure of $\underline{\mathbf{G W}}$-module and the structure of generalized transfers.

REMARK 5.18. In general, it is not true that for a field $\mathcal{I} M(F)$ is just $I(F) \cdot M(F)$. This is true in the case of the sheaves of the form $\underline{\mathbf{I}}^{n}$ for instance by one of the results of $[\mathbf{1}]$.

From what we have seen up to now we get:

Corollary 5.19. Let $M$ be a strictly $\mathbb{A}^{1}$-invariant sheaf $M$ with generalized transfers. Then the quotient sheaf

$$
M / \mathcal{I} M
$$

is in a canonical way a sheaf with transfers in the sense of Voevodsky, and as a consequence has the strong rigidity property.

Proof. By the previous Theorem $M / \mathcal{I} M$ is a strictly $\mathbb{A}^{1}$-invariant sheaf with generalized transfers in which the $\mathbf{G W}$-module structure is trivial. The result follows now from Theorem 5.16.

Proof of TheOrem 5.13. Let $M$ be a strictly $\mathbb{A}^{1}$-invariant sheaf $M$ with generalized transfers. We may assume that $\ell=2$ and that $M$ is a sheaf of $\mathbb{Z} / 2$-modules (by Lemma 5.15). Let $A$ be the (strict) henselization of a geometric point $x$ in a smooth $k$-scheme $X$.

We claim that the filtration $\ldots \mathcal{I}^{n} M(A) \subset \mathcal{I}^{n-1} M(A) \ldots$ is finite. This follows from the previous Theorem, the affirmation by Voevodsky of the Milnor conjectures which implies (see [2, Lemma 2.1]) that for a field $F$ of finite type over its prime field, $I^{n+1}(F)=2 I^{n}(F)$ for $n$ large enough.

Thus to prove that the rigidty property it suffices to check that each of the morphism $I^{n} M / I^{n+1} M(A) \rightarrow I^{n} M / I^{n+1} M(\kappa)$ is an isomorphism, where $\kappa$ is residue field. But this follows from the previous corollary and the classical rigidity property $[\mathbf{6 3}]$.

\section{A $\quad \mathbb{A}^{1}$-coverings and Friedlander conjecture in degree 2}

In this section we give a proof of the Friedlander conjecture in degree 2 . 
A.1. $\mathbb{A}^{1}$-coverings and central extensions of algebraic groups. Recall that $G$ denotes a split semi-simple $k$-group. We want to study a bit the sheaf $\pi_{0}^{\mathbb{A}^{1}}(G)$ defined in [44]. It is clearly a sheaf of groups in the Nisnevich topology.

If moreover $G$ is assumed to be simply connected then for each field extension $F / k$ the group $G(F)$ is generated by the images of the homomorphisms $\mathbb{G}_{a} \rightarrow G$ over $F$, where $\mathbb{G}_{a}$ is the additive group see $[\mathbf{6 5}, \S 1.1 .2]$. Consequently $G$ is weakly 0 -connected and thus $\mathbb{A}^{1}$-connected by $[\mathbf{3 8}$, Lemma 6.1.3], which means that:

Lemma A.1. Let $G$ be a split simply connected semi-simple $k$-group, then

$$
\pi_{0}^{\mathbb{A}^{1}}(G)=*
$$

Now we will use this result to study the $\pi_{0}^{\mathbb{A}^{1}}(G)$ for $G$ an arbitrary split semi-simple $k$-group. Let $T \subset G$ be a split maximal Torus. Let $\tilde{G} \rightarrow G$ be the universal covering in the sense of algebraic groups theory. Let $C \subset \tilde{G}$ be the kernel; it is a finite central abelian group and the morphism a Galois étale covering with Galois group $C$. Let $\tilde{T} \subset \tilde{G}$ be the inverse image of $T$. It is a split maximal torus. Let us denote by $T /{ }_{N i s} \tilde{T}$ the quotient sheaf in the Nisnevich topology. This is an $\mathbb{A}^{1}$-invariant sheaf with transfers in the sense of Voevodsky. The étale short exact sequence $0 \rightarrow C \rightarrow \tilde{T} \rightarrow T \rightarrow 0$ and Hilbert Theorem 90 ( $\tilde{T}$ being also split) gives an isomorphism of sheaves in the Nisnevich topology

$$
T /{ }_{N i s} \tilde{T} \rightarrow \mathcal{H}_{e t}^{1}(C)
$$

where $\mathcal{H}_{e t}^{1}(C)$ is the associated sheaf to $X \mapsto H_{e t}^{1}(X ; C)$.

TheOREM A.2. Keeping the above notations and assumptions, the morphism of sheaves $T \rightarrow \pi_{0}^{\mathbb{A}^{1}}(G)$ induced by the inclusion $T \subset G$ is onto and induces an isomorphism

$$
T /{ }_{\text {Nis }} \tilde{T} \cong \pi_{0}^{\mathbb{A}^{1}}(G)
$$

Moreover this sheaf clearly vanishes in the étale topology.

Proof. It follows from the following commutative diagram of smooth $k$-schemes

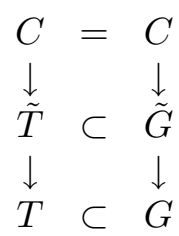

whose bottom square is cartesian. First it induces a isomorphism of quotient smooth $k$-schemes $\tilde{G} / \tilde{T} \cong G / T$. Now by the previous Theorem, $\tilde{G}$ being simply connected (in the sense of algebraic group theory) is $\mathbb{A}^{1}$-connected. But the morphism $\tilde{G} \rightarrow \tilde{G} / \tilde{T}$ is Zariski (thus Nisnevich) locally trivial and induces an isomorphism on the $\pi_{0}^{\mathbb{A}^{1}}$. Thus $G / T$ is $\mathbb{A}^{1}$-connected. 
As $\tilde{G} \rightarrow \tilde{G} / \tilde{T}$ and $G \rightarrow G / T$ are $\mathbb{A}^{1}$-coverings (being a Zariski locally trivial $T$-torsor, with $T$ split) $[41]$ we get long exact sequences of $\mathbb{A}^{1}$-homotopy sheaves.

The one for $G \rightarrow G / T$ gives an exact sequence (in the Nisnevich topology) of the form

$$
\pi_{1}^{\mathbb{A}^{1}}(G / T) \rightarrow T \rightarrow \pi_{0}^{\mathbb{A}^{1}}(G) \rightarrow 0
$$

and the second one gives that $\pi_{1}^{\mathbb{A}^{1}}(G / T) \cong \pi_{1}^{\mathbb{A}^{1}}(\tilde{G} / \tilde{T}) \rightarrow \tilde{T} \rightarrow \pi_{0}^{\mathbb{A}^{1}}(\tilde{G})=0$ is onto. This clearly implies the claim.

REMARK A.3. In the case $G=S O_{n}^{\prime}$ (split) for instance the quotient sheaf $T /{ }_{N i s} T^{\prime}$ is isomorphic to the sheaf $\mathbb{G}_{m} /\left(\mathbb{G}_{m}\right)^{2}$ and the morphism $S O_{n}^{\prime} \rightarrow$ $\pi_{0}^{\mathbb{A}^{1}}\left(S O_{n}^{\prime}\right)=\mathbb{G}_{m} /\left(\mathbb{G}_{m}\right)^{2}$ is the spinor norm.

Let $G^{(0)} \subset G$ be the kernel of $G \rightarrow \pi_{0}^{\mathbb{A}^{1}}(G)$.

LEMmA A.4. The induced morphism $\tilde{G} \rightarrow G^{(0)}$ is an epimorphism in the Nisnevich topology.

Proof. One use the short exact sequence in the étale topology $0 \rightarrow$ $C \rightarrow \tilde{G} \rightarrow G$ to get for any smooth $F$-scheme $X$ an associated long exact of cohomology groups/sets

$$
\tilde{G}(X) \rightarrow G(X) \rightarrow H_{e t}^{1}(X ; C) \rightarrow \cdots
$$

by sheafifying in the Nisnevich topology we get the exact sequence:

$$
\tilde{G} \rightarrow G \rightarrow \mathcal{H}_{e t}^{1}(C)
$$

which easily implie the claim taking the above isomorphism $\pi_{0}^{\mathbb{A}^{1}}(G) \cong$ $\mathcal{H}_{\text {et }}^{1}(C)$ into account.

By construction $G^{(0)}$ is an $\mathbb{A}^{1}$-connected space with a base point (the neutral element). Thus it admits by $[\mathbf{4 1}]$ a universal $\mathbb{A}^{1}$-covering $\tilde{G}^{\mathbb{A}^{1}} \rightarrow G^{(0)}$ which is easily seen as usual to define a central extension in the Nisnevich topology of the form

$$
0 \rightarrow \pi_{1}^{\mathbb{A}^{1}}(G) \rightarrow \tilde{G}^{\mathbb{A}^{1}} \rightarrow G^{(0)} \rightarrow 1
$$

If $G$ is simply connected, it follows from the previous Theorem that $\pi_{0}^{\mathbb{A}^{1}}(G)=*$ and that the previous extension gives a central extension in the Nisnevich topology ${ }^{8}$ of the form

$$
0 \rightarrow \pi_{1}^{\mathbb{A}^{1}}(G) \rightarrow \tilde{G}^{\mathbb{A}^{1}} \rightarrow G \rightarrow 1
$$

Now for $G$ general (non necessarily simply connected) we see that the associated morphism of sheaves in the étale topology to $G^{(0)} \rightarrow G$ is an isomorphism as this follows from Theorem A.2 above. Consequently the

\footnotetext{
8 actually in the Zariski topology as well
} 
associated central extension to (A.1) in the étale topology has the following form

$$
0 \rightarrow \pi_{1}^{\mathbb{A}^{1}}(G)_{e t} \rightarrow \tilde{G}_{e t}^{\mathbb{A}^{1}} \rightarrow G \rightarrow 1
$$

This extension has the following universal property:

Theorem A.5. Given a central extension in the étale topology

$$
0 \rightarrow M \rightarrow E \rightarrow G \rightarrow 1
$$

such that $M$ is a locally constant étale sheaf of torsion prime to char $(k)$, then there exists one and only one morphism of sheaves in the étale topology $\pi_{1}^{\mathbb{A}^{1}}(G)_{\text {et }} \rightarrow M$ which induces the given extension from (A.3). In other words:

$$
H_{e t}^{2}(G ; M) \cong \operatorname{Hom}_{\mathcal{A} b(k)}\left(\pi_{1}^{\mathbb{A}^{1}}(G), M\right)
$$

where $\mathcal{A} b(k)$ means the category of sheaves of abelian groups in the Nisnevich topology on $\mathrm{Sm}_{k}$.

Proof. Given a central extension in the étale topology $0 \rightarrow M \rightarrow$ $E \rightarrow G \rightarrow 1$ with $M$ as above, the morphism $E \rightarrow G$ is an $\mathbb{A}^{1}$-covering [41]. From the universal property of the universal $\mathbb{A}^{1}$-covering, we get a morphism $\bar{G}^{\mathbb{A}^{1}} \rightarrow E$; the associated morphism in the étale topology has the required property. Uniqueness is easy to establish along the same lines.

REMARK A.6. The previous Theorem can be strenghened as follows: say that a sheaf of abelian groups $M$ is the étale topology is strictly étale $\mathbb{A}^{1}$-invariant if $X \mapsto H_{e t}^{i}(X ; M)$ is $\mathbb{A}^{1}$-invariant for any $i \in \mathbb{N}$. For instance any $\mathbb{A}^{1}$-invariant étale sheaf of $\mathbb{Z}\left[\frac{1}{p}\right]$-modules $^{9}$ with transfers in the sense of Voevodsky is strictly étale $\mathbb{A}^{1}$-invariant by $[\mathbf{6 8}$, Corollary 5.29 p.136]. Then one may prove that $\pi_{1}^{\mathbb{A}^{1}}(G)\left[\frac{1}{p}\right]_{e t}$ is srongly étale $\mathbb{A}^{1}$-invariant and that the "induced" central extension

$$
0 \rightarrow \pi_{1}^{\mathbb{A}^{1}}(G)\left[\frac{1}{p}\right]_{e t} \rightarrow \tilde{G}_{e t}^{\prime} \rightarrow G \rightarrow 1
$$

is the universal central extension with kernel a strictly étale $\mathbb{A}^{1}$-invariant sheaf of $\mathbb{Z}\left[\frac{1}{p}\right]$-modules.

A.2. Proof of the Friedlander conjecture in degree 2. In this section we give a proof of the Friedlander conjecture in degree 2 using the construction of the previous section. Using the classical devissage and reductions, we see that it suffices to check the case $G$ is simple and simply connected (in the sense of algebraic group theory), and $F$ is separably closed.

The left hand side of the Friedlander conjecture is the group $H_{e t}^{2}(B G$; $\mathbb{Z} / \ell)$ that is to say the group of isomorphism classes of central extensions

$$
0 \rightarrow \mathbb{Z} / \ell \rightarrow E \rightarrow G \rightarrow 1
$$

\footnotetext{
${ }^{9}$ here $p$ is the characteristic exponent of $F$
} 
of sheaves of groups in the étale topology. From the Theorem A.5, this group is isomorphic to $\operatorname{Hom}_{\mathcal{A b}(k)}\left(\pi_{1}^{\mathbb{A}^{1}}(G), \mathbb{Z} / \ell\right)$.

On the other hands, the right hand side is the group $H^{2}(B G(F) ; \mathbb{Z} / \ell)$ of isomorphism classes of central extensions

$$
0 \rightarrow \mathbb{Z} / \ell \rightarrow E \rightarrow G(F) \rightarrow 1
$$

The group $G(F)$ is known to be perfect, $F$ being infinite, so it admits a universal central extension of the form ${ }^{10}$

$$
0 \rightarrow H_{2}(B G(F)) \rightarrow \widetilde{G(F)}^{a l g} \rightarrow G(F) \rightarrow 1
$$

and $H^{2}(B G(F) ; \mathbb{Z} / \ell) \cong H o m\left(H_{2}(B G(F), \mathbb{Z} / \ell)\right.$. We now claim the following, which establish the Friedlander conjecture in degree 2.

Lemma A.7. With $G$ as above and $F$ separably closed,

$$
\operatorname{Hom}\left(H_{2}(B G(F), \mathbb{Z} / \ell)=\operatorname{Hom}_{\mathcal{A} b(k)}\left(\pi_{1}^{\mathbb{A}^{1}}(G), \mathbb{Z} / \ell\right)=0\right.
$$

Proof. Hom $\left(H_{2}(B G(F)), \mathbb{Z} / \ell\right)=0$ follows from the classical computations of Matsumoto [33] which shows that $H_{2}(B G(F)) \cong K_{2}^{M}(F)$ and thus is $\ell$-divisible. The vanishing of $\operatorname{Hom}_{\mathcal{A} b(k)}\left(\pi_{1}^{\mathbb{A}^{1}}(G), \mathbb{Z} / \ell\right)$ follows from the fact that the inclusion $S L_{2} \rightarrow G$ coming from a root of maximal length induces an epimorphism of sheaves (in the Nisnevich topology):

$$
\underline{\mathbf{K}}_{2}^{M W} \cong \pi_{1}^{\mathbb{A}^{1}}\left(S L_{2}\right) \rightarrow \pi_{1}^{\mathbb{A}^{1}}(G)
$$

This can be done using for instance [57, Theorem 4.1].

REMARK A.8. It can be shown that for $G$ a split simply connected absolutely simple $F$-group, $F$ arbitrary, then the previous epimorphism $K_{2}^{M W} \cong \pi_{1}^{\mathbb{A}^{1}}\left(S L_{2}\right) \rightarrow \pi_{1}^{\mathbb{A}^{1}}(G)$ induces an isomorphism of sheaves

$$
\underline{\mathbf{K}}_{2}^{M} \cong \pi_{1}^{\mathbb{A}^{1}}(G)
$$

except when $G$ is of symplectic type, in which case

$$
\underline{\mathbf{K}}_{2}^{M W} \cong \pi_{1}^{\mathbb{A}^{1}}(G)
$$

is an isomorphism. Also it can be shown that the central extension

$$
0 \rightarrow \underline{\mathbf{K}}_{2}^{M} \rightarrow E \rightarrow G \rightarrow 1
$$

induced by (A.2) and the epimorphism $K_{2}^{M W} \rightarrow \pi_{1}^{\mathbb{A}^{1}}(G)$ is the one constructed [6], at least for $F$ infinite.

Evaluting the universal extension (A.2) on $F$ and comparing with the previous one of $G(F)$, we get a canonical homomorphism $H_{2}(B G(F)) \rightarrow$

\footnotetext{
$10_{\text {to avoid }}$ notational contradictions, we choosed to write $H_{2}(B G(F))$ for the group which is often denoted by $H_{2}(G(F))$
} 
$\pi_{1}^{\mathbb{A}^{1}}(G)(F)$. It is also possible to show in fact that it is always an isomorphism (except maybe for very small finite fields). In other words we get an isomorphism, for any infinite field $F$

$$
H_{2}(B G(F)) \cong H_{2}^{\mathbb{A}^{1}}(B G)(F)=\pi_{1}^{\mathbb{A}^{1}}(G)(F)
$$

This is a stronger result as the Friedlander conjecture in degree 2.

\section{References}

[1] J. Arason, Cohomologische invarianten quadratischer Formen. (German) J. Algebra 36 (1975), no. 3, 448-491.

[2] J. Arason and R. Elman, Powers of the Fundamental Ideal in the Witt Ring, J. Algebra 239 (2001).

[3] M. Artin and B. Mazur, Étale Homotopy, Lect. Notes in Maths 100, Springer Heidelberg 1969.

[4] N. Bourbaki, Groupes et algèbres de Lie, Chapitre 1.

[5] A.K. Bousfield and D.M. Kan, Homotopy limits, completions and localizations. Lecture Notes in Mathematics, Vol. 304. Springer-Verlag, Berlin-New York, 1972.

[6] J.-L. Brylinski and P. Deligne, Central extensions of reductive groups by $\mathbf{K}_{2}$. Publ. Math. Inst. Hautes tudes Sci. No. 94 (2001), 5-85.

[7] Séminaire Henri Cartan, 7 no. 1, 1954-1955, Algbre d'Eilenberg-Maclane et homotopie.

[8] E. Curtis, Some relations between homotopy and homology, Ann. of Maths. 83 (1965), $159-171$

[9] E. Curtis, simplicial homotopy theory, Advances in Maths.

[10] A. Dold, Homology of Symmetric Products and Other Functors of Complexes, Annals of Math.. 68 (1958), 54-80.

[11] A. Dold, Les foncteurs dérivés d'un foncteur non-additif. Séminaire Bourbaki, 5 (1958-1960), Exposé No. 170.

[12] E. Friedlander, The Friedlander-Milnor conjecture, L'enseignement Mathématique, (2) 54, 2008, 90-92.

[13] E. Friedlander, Étale homotopy of simplicial schemes, Ann. of Math. Sudies, PUP.

[14] E. Friedlander and G. Mislin, Cohomology of classifying spaces of complex Lie groups and related discrete groups. Comment. Math. Helv. 59 (1984), no. 3, 347-361.

[15] O. Gabber, K-theory of Henselian local rings and Henselian pairs, Contemp. Math. 126 (1992), 59-70.

[16] O. Gabber, Gersten's conjecture for some complexes of vanishing cycles. Manuscripta Math. 85 (1994), no. 3-4, 323-343.

[17] S.M. Gersten, Higher K-theory of rings, LNM 341.

[18] H. Gillet and R. Thomason, The K-theory of strict hensel local rings and a theorem of Suslin, J. Pure Appl. Algebra 34 (1984), 241-254.

[19] A. Grothendieck and J. Dieudonné, Éléments de Géométrie Algébrique IV. Étude locale des schémas et des morphismes de schémas (Seconde Partie). (French) Inst. Hautes Études Sci. Publ. Math. No. 24, 1965.

[20] A. Grothendieck and J. Dieudonné, Éléments de Géométrie Algébrique IV. Étude locale des schémas et des morphismes de schémas (Troisième Partie). (French) Inst. Hautes Études Sci. Publ. Math. No. 28, 1967, 361 pp.

[21] A. Grothendieck and J. Dieudonné, Éléments de Géométrie Algébrique IV. Étude locale des schémas et des morphismes de schémas (Quatrième Partie). (French) Inst. Hautes Études Sci. Publ. Math. No. 32, 1967.

[22] A. Grothendieck, Séminaire de Géométrie Algébrique, Revêtements étales et Groupe Fondamental, L.N.M. 224. 
[23] A. Grothendieck, Séminaire de Géométrie Algébrique, Théorie des Topos et cohomologie étale des schémas (SGA 4), Lect. Notes in Maths 269,270, et 305, Springer Heidelberg, 1972-73.

[24] F. Grunewald, J. Mennicke and L. Vaserstein: On the groups $\mathrm{SL}_{2}(\mathbf{Z}[x])$ and $\mathrm{SL}_{2}(k[x, y])$. Israel J. Math. 86 (1994), no. 1-3, 157-193.

[25] R. Hartschorne, Algebraic geometry, Springer 1977.

[26] M. Hovey, Model category structures on chain complexes of sheaves, Transactions of the AMS 353 (2001), 2441-2457.

[27] J. F. Jardine, On the homotopy groups of algebraic groups. J. Algebra 81 (1983), no. $1,180-201$.

[28] J. F. Jardine, Simplicial objects in a Grothendieck topos, Contemp. Math. 55 (1986), 193-239.

[29] M. Karoubi, Relations between algebraic K-theory and Hermitian K-theory. Journal of Pure and Applied Algebra 34, p. 259-263 (1984).

[30] K. Knudson, Homology of linear groups, Birkhäuser, Basel-Boston-Berlin.

[31] K. Knudson, The homology of special linear groups over polynomial rings, Ann. Sci. École Norm. Sup. (4) 30 (1997), no 3, 385-415.

[32] T.Y. Lam, Serre's conjecture, L.N.M. 635.

[33] H. Matsumoto, Sur les sous-groupes arithmétiques des groupes semi-simples déployés. (French) Ann. Sci. École Norm. Sup. (4) 2 1969, 1-62.

[34] J. Milne, Étale cohomology, Princeton University Press.

[35] J. Milnor, On the homology of Lie groups made discrete, Comment. Math. Helvetici 58 (1953) 072-085.

[36] J. Milnor, D. Husemoller, Symmetric bilinear forms, Ergebnisse der Mathematik, Band 73, Springer-Verlag.

[37] F. Morel, Sur les puissances de l'idéal fondamental de l'anneau de Witt, Commentarii Mathematici Helvetici, Vol 79 no 4, (2004) p 689.

[38] F. Morel, The stable $\mathbb{A}^{1}$-connectivity theorems, K-theory (2005) 35:p.1-68.

[39] F. Morel, $\mathbb{A}^{1}$-algebraic topology, Proceedings of the International Congress of Mathematicians, Madrid 2006.

[40] F. Morel, Milnor's conjecture on quadratic forms and mod 2 motivic complexes, Rend. Sem. Mat. Univ. Padova, Vol 114 (2005) pp 63-101.

[41] F. Morel, $\mathbb{A}^{1}$-algebraic topology over a field, preprint (2011).

[42] F. Morel, The weak Friedlander-Milnor conjecture, preprint (2010).

[43] F. Morel, Generalized transfers and the rigidity property for the $C_{*}^{\mathbb{A}^{1}}\left(\left(\mathbb{G}_{m}\right)^{\wedge n}\right)$ 's, in preparation.

[44] F. Morel and V. Voevodsky, $\mathbb{A}^{1}$-homotopy theory of schemes, Publications Mathématiques de l'I.H.É.S. no 90.

[45] L.-F. Moser, $\mathbb{A}^{1}$-locality results for linear algebraic groups, in preparation.

[46] M. Pavaman Murthy, Zero cycles and projective modules, Ann. of Maths, 140 (1994).

[47] Y. Nisnevich, The completely decomposed topology on schemes and associated descent spectral sequences in algebraic $K$-theory. Algebraic $K$-theory: connections with geometry and topology (Lake Louise, AB, 1987), 241-342, NATO Adv. Sci. Inst. Ser. C Math. Phys. Sci., 279, Kluwer Acad. Publ., Dordrecht, 1989.

[48] D. Orlov, A. Vishik and V. Voevodsky, An exact sequence for $\mathrm{K}^{*} \mathrm{M} / 2$ with applications to quadratic forms Annals of Mathematics, 165(1), 1-13. 2007.

[49] D. Quillen, Homotopical algebra, L.N.M.

[50] C-H. Sah, Homology of classical Lie groups made discrete. III. J. Pure Appl. Algebra 56 (1989), no. 3, 269-312.

[51] J.W. Schlesinger, The semi-simplicial free Lie ring, Trans. Amer. Math. Soc. 122 (1966), 436-442. 
[52] W. Scharlau, Quadratic and Hermitian forms. Grundlehren der Mathematischen Wissenschaften [Fundamental Principles of Mathematical Sciences], 270. Springer-Verlag, Berlin, 1985.

[53] Schmid, Wittring Homology, Phd Dissertation, Universitaet Regensburg, 1998.

[54] J.-P. Serre, Corps locaux, Hermann, Paris 1968.

[55] T. Springer, Linear algebraic groups. Progress in Mathematics, Volume 9, Birkhäuser.

[56] Soulé, Christophe Chevalley groups over polynomial rings. Homological group theory (Proc. Sympos., Durham, 1977), pp. 359-367, London Math. Soc. Lecture Note Ser., 36, Cambridge Univ. Press, Cambridge-New York, 1979.

[57] M. Stein, Chevalley groups over commutative rings. Bull. Amer. Math. Soc. 77 (1971), $247-252$.

[58] D. Sullivan, Genetics of homotopy theory and the Adams conjecture, Ann. of Math, 100.

[59] A. Suslin, The structure of the special linear group over rings of polynomials. (Russian) Izv. Akad. Nauk SSSR Ser. Mat. 41 (1977), no. 2, 235-252, 477.

[60] A. Suslin, On the $K$-theory of algebraically closed fields, Invent. Math. 73 (1983), no. $2,241-245$.

[61] A. Suslin, On the $K$-theory of local fields. Proceedings of the Luminy conference on algebraic $K$-theory (Luminy, 1983). J. Pure Appl. Algebra 34 (1984), no. 2-3, 301-318.

[62] A. Suslin, Homology of $\mathrm{GL}_{n}$, characteristic classes and Milnor $K$-theory. Algebraic $K$-theory, number theory, geometry and analysis (Bielefeld, 1982), 357-375, Lecture Notes in Math., 1046, Springer, Berlin, 1984.

[63] A. Suslin and V. Voevodsky, Singular homology of abstract algebraic varieties, Invent. Math. 123 (1996) 6394.

[64] A. Suslin and V. Voevodsky, Relative cycles and Chow sheaves, Cycles, transfers, and motivic homology theories, 188-238, Ann. of Math. Stud., 143, Princeton Univ. Press, Princeton, NJ, 2000.

[65] J. Tits, Groupes de Whitehead de groupes algébriques simples sur un corps, Séminaire Bourbaki, Exposé No. 505, 1976/77.

[66] V. Voevodsky. Motivic Cohomology with Z/2-coefficients, Inst. Hautes Études Sci. Publ. Math., vol. 98 (2003), 59-104.

[67] V. Voevodsky, Triangulated categories of motives over a field. Cycles, transfers, and motivic homology theories, 188-238, Ann. of Math. Stud., 143, Princeton Univ. Press, Princeton, NJ, 2000.

[68] V. Voevodsky, Cohomological Theory of Presheaves with Transfers. Cycles, transfers, and motivic homology theories, 87-137, Ann. of Math. Stud., 143, Princeton Univ. Press, Princeton, NJ, 2000.

[69] M. Wendt, $\mathbb{A}^{1}$-homotopy of Chevalley groups, J. K-Theory 5 (2), 2010, pp. 245-287.

[70] M. Wendt, Homotopy Invariance for Homology of Linear Groups: Reductions and the rank two case. Preprint. Available at http://arxiv.org/abs/1011.5110.

[71] M. Wendt, Homotopy Invariance for Homology of Linear Groups: The Case SL4. Preprint. Available at http://arxiv.org/abs/1011.5392. 
